TRANSACTIONS OF THE

AMERICAN MATHEMATICAL SOCIETY

Volume 355, Number 5, Pages 2139-2160

S 0002-9947(03)03251-3

Article electronically published on January 14, 2003

\title{
LOCAL POWER SERIES QUOTIENTS OF COMMUTATIVE BANACH AND FRÉCHET ALGEBRAS
}

\author{
MARC P. THOMAS
}

\begin{abstract}
We consider the relationship between derivations and local power series quotients for a locally multiplicatively convex Fréchet algebra. In $\S 2$ we derive necessary conditions for a commutative Fréchet algebra to have a local power series quotient. Our main result here is Proposition 2.6, which shows that if the generating element has finite closed descent, the algebra cannot be simply a radical algebra with identity adjoined - it must have nontrivial representation theory; if the generating element does not have finite closed descent, then the algebra cannot be a Banach algebra, and the generating element must be locally nilpotent (but non-nilpotent) in an associated quotient algebra. In $\S 3$ we consider a fundamental situation which leads to local power series quotients. Let $D$ be a derivation on a commutative radical Fréchet algebra $\mathcal{R}^{\sharp}$ with identity adjoined. We show in Theorem 3.10 that if the discontinuity of $D$ is not concentrated in the (Jacobson) radical, then $\mathcal{R}^{\sharp}$ has a local power series quotient. The question of whether such a derivation can have a separating ideal so large it actually contains the identity element has been recently settled in the affirmative by C. J. Read.
\end{abstract}

\section{$\S 1$. INTRODUCTION}

The Singer-Wermer conjecture states that a (possibly discontinuous) derivation on a commutative Banach algebra maps into the (Jacobson) radical. In establishing a proof of this conjecture one first uses reductions of B. E. Johnson [Johnson] and Thomas Thomas2 to show that if the conjecture fails, then some commutative radical Banach algebra $\mathcal{A}$ has an element $x$ with finite closed descent (see Definition 1.10) and a formal power series quotient based at that $x$ (see Definition 2.1). Such a quotient is then shown to be impossible by constructing a finite subset called a recalcitrant system (see [Thomas2, Definition 3.3]). Recalcitrant systems essentially preclude prime-like properties, but they seem to require either finite closed descent or a related condition such as $a^{n} \in \overline{a^{n} \mathcal{A}}$ for construction.

Much the same strategy can be used to analyze a derivation on a locally multiplicatively convex Fréchet algebra. What is surprising is that the conditions necessary for the existence of a formal power series quotient are not greatly different whether one considers a Banach or Fréchet algebra (see Proposition 2.6), although

Received by the editors August 27, 2001 and, in revised form, October 18, 2002.

2000 Mathematics Subject Classification. Primary 46H05.

The author thanks Pomona College for support as a Visiting Scholar during the summer of the Banach Algebras 1999 conference and the Centre for Mathematics and its Applications for support during the Banach Spaces, Operators, and Algebras Symposium in January 2001 at the Australian National University. 
such quotients are far more prevalent in Fréchet algebras where it is possible to have locally nilpotent, non-nilpotent, elements.

The Fréchet and Banach algebras in this paper will generally be commutative. Commutativity is essential in Section 2 since the kernel of $\theta$ (see Definition 2.1) needs to be an intersection of range spaces. The noncommutative version of Definition 2.1 requires handling the ideal generated by the commutators; this is probably one contributing reason why the noncommutative Singer-Wermer conjecture has remained unproven.

Throughout this paper we use the terms algebra, ideal, and subspace, in the algebraic sense; that is, we do not assume that these substructures are closed.

In this paper $\mathcal{A}$ will denote a Fréchet algebra with topology given by an increasing sequence of multiplicatively convex seminorms $\|\cdot\|_{n}$ in the usual way.

If $\mathcal{A}$ does not have an identity element, we extend each of these seminorms to $\mathcal{A}^{\sharp}$ in the usual way; that is,

$$
\|\lambda 1+a\|_{n} \equiv|\lambda|+\|a\|_{n}
$$

for $\lambda \in \mathbb{C}$ and $a \in \mathcal{A}$. We can then let $\mathcal{I}_{n}=\left\{a \in \mathcal{A}^{\sharp} \mid\|a\|_{n}=0\right\}$ and let $\mathcal{B}_{n}$ denote the completion of $\left(\mathcal{A}^{\sharp} / \mathcal{I}_{n},\|\cdot\|_{n}\right)$. We will let $\pi_{n}$ denote the natural map from $\mathcal{A}^{\sharp}$ into $\mathcal{B}_{n}$. We note in passing that Michael's Theorem [Michael, Theorem 5.1] gives an explicit realization of $\mathcal{A}^{\sharp}$ in terms of a projective limit of the $\mathcal{B}_{n}$ 's.

We now require some standard definitions concerning nilpotency, spectrum, and torsion.

Definition 1.1. Let $\mathcal{A}$ be a Fréchet algebra. We say that an element $a \in \mathcal{A}$ is nilpotent if there exists $n \in \mathbf{N}$ such that $a^{n}=0$. We say that $a$ is locally nilpotent if for each $N \in \mathbf{N}$ there is $n \in \mathbf{N}$ such that $\left\|a^{n}\right\|_{N}=0$. If this is not the case, there must exist some $N \in \mathbf{N}$ such that $\left\|a^{n}\right\|_{N}>0$ for all $n \in \mathbf{N}$ and we say that $a$ is nonlocally nilpotent.

If $\mathcal{A}$ is actually a Banach algebra, local nilpotency and nilpotency are equivalent.

Definition 1.2. We define the spectrum of an element $a \in \mathcal{A}$ in the usual way:

$$
\sigma(a) \equiv\left\{\lambda \in \mathbb{C} \mid(\lambda 1-a) \text { is not invertible in } \mathcal{A}^{\sharp}\right\} ;
$$

and define the spectral radius in the usual way:

$$
\rho_{\sigma}(a) \equiv \sup \{|\lambda| \mid \lambda \in \sigma(a)\} .
$$

Since the spectrum in a Fréchet algebra (unlike the case of a Banach algebra) need not be compact, we allow $\rho_{\sigma}(a)$ to take the value $+\infty$.

Definition 1.3. Let $\mathcal{A}$ be a commutative Fréchet algebra and let $a \in \mathcal{A}$. For each $n \in \mathbf{N}$ the element $\pi_{n}(a)$ is an element in the Banach algebra $\mathcal{B}_{n}$ so that $\sigma\left(\pi_{n}(a)\right)$ is a nonempty compact subset of the complex plane. For ease of notation we denote $\sigma_{n}(a)=\sigma\left(\pi_{n}(a)\right)$ for all $n \in \mathbf{N}$.

It is routine to check that $\sigma_{n}(a) \subseteq \sigma_{n+1}(a)$ and that

$$
\max \left\{|\lambda| \mid \lambda \in \sigma_{n}(a)\right\}=\lim _{i \rightarrow \infty}\left\|\pi_{n}\left(a^{i}\right)\right\|_{\mathcal{B}_{n}}^{1 / i} \leq\left\|\pi_{n}(a)\right\|_{\mathcal{B}_{n}}=\|a\|_{n}
$$

for all $n \in \mathbf{N}$. It is also the case that $\sigma(a)=\bigcup_{n=1}^{\infty} \sigma_{n}(a)$ (see Michael, Theorem $5.3])$. 
Definition 1.4. Let $\mathcal{A}$ be a commutative Fréchet algebra and fix an element $a \in \mathcal{A}$. We define the set of torsion elements (with respect to $a$ ) to be

$$
\mathcal{T}(a) \equiv\left\{v \in \mathcal{A}^{\sharp} \mid a^{n} v=0 \text { for some } n \in \mathbf{N}\right\} .
$$

It is routine to check that the torsion elements form an ideal of $\mathcal{A}^{\sharp}$ (which is generally not closed).

Definition 1.5. Let $\mathcal{A}$ be a commutative Fréchet algebra and fix an element $a \in \mathcal{A}$. We define the height of an element $v$ (with respect to $a$ ) to be

$$
\begin{gathered}
h(v)=0 \text { if } v \notin a \mathcal{A}^{\sharp}, \\
h(v)=n \text { if } v \in a^{n} \mathcal{A}^{\sharp}-a^{n+1} \mathcal{A}^{\sharp},
\end{gathered}
$$

and

$$
h(v)=\infty \text { if } v \in \bigcap_{n=1}^{\infty} a^{n} \mathcal{A}^{\sharp} .
$$

We note that since $a^{n+1} \mathcal{A}^{\sharp} \subseteq a^{n} \mathcal{A}$, the set $\bigcap_{n=1}^{\infty} a^{n} \mathcal{A}^{\sharp}$ of elements of infinite height is also equal to $\bigcap_{n=1}^{\infty} a^{n} \mathcal{A}$. It is routine to check that the set of elements of infinite height forms an ideal, but one important feature of this ideal is that it has a Fréchet topology $\tau_{h}$ which is (formally) stronger than the original Fréchet topology $\tau$ on $\mathcal{A}$, namely the topology generated from the range space topology on each $a^{n} \mathcal{A}$. A sequence $\left\{v_{i}\right\}_{i=1}^{\infty}$ converges in $\bigcap_{n=1}^{\infty} a^{n} \mathcal{A}$ if and only if for each $n \in \mathbf{N}$ it is the image (under multiplication by $a^{n}$ ) of a convergent sequence in $\mathcal{A}$.

There is a relationship between torsion elements and divisibility.

Definition 1.6. Let $\mathcal{A}$ be a Fréchet algebra and fix an element $a \in \mathcal{A}$. We say that a subspace $H$ of $\mathcal{A}$ is a-divisible if for every $\lambda \in \mathbb{C}$ we have $(\lambda-a) H=H$ (if $\mathcal{A}$ is noncommutative, this is usually qualified as "left-divisible"). There is always a largest $a$-divisible subspace, which we will denote $\mathcal{D}_{a}$ (which might be $\{0\}$ ).

Lemma 1.7. Let $\mathcal{A}$ be a commutative Fréchet algebra and let $a \in \mathcal{A}$ satisfy $\mathcal{T}(a) \subseteq$ $a \mathcal{A}$. Then

(i) the ideal of torsion elements $\mathcal{T}(a)$ is a-divisible,

(ii) $a\left(\bigcap_{n=1}^{\infty} a^{n} \mathcal{A}\right)=\left(\bigcap_{n=1}^{\infty} a^{n} \mathcal{A}\right)$.

Proof. First note that $\mathcal{T}(a)$ is closed under division by $a$; that is, if $a w=v$, then $v \in \mathcal{T}(a)$ implies that $w \in \mathcal{T}(a)$. This shows that $a \mathcal{T}(a)=\mathcal{T}(a)$. Let $\lambda \in \mathbb{C}$ be nonzero and let $v \in \mathcal{T}(a)$. There exists $n \in \mathbf{N}$ such that $a^{n} v=0$. Choose polynomials $p$ and $q$ so that $p(x)(\lambda-x)+q(x) x^{n}=1$. Let $x=a$ and apply this polynomial equation to $v$,

$$
v=p(a)(\lambda-a) v+q(a) a^{n} v=(\lambda-a)(p(a) v) .
$$

This shows that $(\lambda-a) \mathcal{T}(a)=\mathcal{T}(a)$ for all $\lambda \neq 0$, completing the proof of (i). Now let $v \in\left(\bigcap_{n=1}^{\infty} a^{n} \mathcal{A}\right)$. Hence there exists $v_{n} \in \mathcal{A}$ such that $a^{n} v_{n}=v$ for all $n \in \mathbf{N}$. It suffices to show that $v_{1} \in\left(\bigcap_{n=1}^{\infty} a^{n} \mathcal{A}\right)$. But, $\left(a^{n-1} v_{n}-v_{1}\right) \in \mathcal{T}(a)$. Since $\mathcal{T}(a)$ is $a$-divisible, we can find $w_{n} \in \mathcal{T}(a)$ such that $a^{n-1} w_{n}=a^{n-1} v_{n}-v_{1}$ for all $n \in \mathbf{N}$. This shows that $v_{1} \in\left(\bigcap_{n=1}^{\infty} a^{n-1} \mathcal{A}\right)=\left(\bigcap_{n=1}^{\infty} a^{n} \mathcal{A}\right)$, ending the proof of (ii).

We will have to handle one special case involving closed range when $\mathcal{A}$ is a Banach algebra in the next section. 
Lemma 1.8. Let $\mathcal{A}$ be a commutative Banach algebra. Let $a \in \mathcal{A}$ satisfy $a \mathcal{A}=\overline{a \mathcal{A}}$ and $\mathcal{T}(a) \subseteq a \mathcal{A}$. There exists a constant $K$ such that whenever $n \in \mathbf{N}$ and $\left\{a^{n} e_{i}\right\}_{i=1}^{\infty}$ is a sequence satisfying $\left\|a^{n} e_{i}-a^{n} e_{i+1}\right\|<2^{-i}$ for all $i \in \mathbf{N}$, we can find a new sequence $\left\{a^{n-1} f_{i}\right\}_{i=1}^{\infty}$ satisfying

(i) $a\left(a^{n-1} f_{i}\right)=a^{n} e_{i}$ for all $i \in \mathbf{N}$,

(ii) $\left\|a^{n-1} f_{i}-a^{n-1} f_{i+1}\right\|<K 2^{-i}$ for all $i \in \mathbf{N}$.

Proof. Since the range of the multiplication operator $M_{a}$ on $\mathcal{A}$ is closed, the open mapping theorem guarantees the existence of a constant $K$ such that whenever $x=a y$ we can find $z$ such that $x=a z$ with $\|z\| \leq K\|x\|$ and $(y-z) \in \mathcal{N}_{1} \equiv$ $\{w \in \mathcal{A} \mid a w=0\} \subseteq \mathcal{T}(a)$. We also have that $a \mathcal{T}(a)=\mathcal{T}(a)$ by Lemma 1.7.

Start the induction by letting $f_{1}=e_{1}$ so that $a\left(a^{n-1} f_{1}\right)=a^{n} e_{1}$ and $\| a^{n} f_{1}-$ $a^{n} e_{2} \|<2^{-1}$. By our above remarks we can find $y \in \mathcal{A}$ such that $a y=\left(a^{n} f_{1}-a^{n} e_{2}\right)$ and $\|y\|<K 2^{-1}$, with $y-\left(a^{n-1} f_{1}-a^{n-1} e_{2}\right)=h_{2} \in \mathcal{N}_{1} \subseteq \mathcal{T}(a)$. Since $\mathcal{T}(a)$ is $a$-divisible we can find $g_{2} \in \mathcal{T}(a)$ such that $a^{n-1} g_{2}=h_{2}$ and, hence, $a^{n} g_{2}=0$. Let $f_{2}=e_{2}-g_{2}$ and compute

$$
\begin{gathered}
a\left(a^{n-1} f_{2}\right)=a^{n} e_{2}-a^{n} g_{2}=a^{n} e_{2} \\
\left\|a^{n-1} f_{1}-a^{n-1} f_{2}\right\|=\left\|a^{n-1} f_{1}-a^{n-1} e_{2}+a^{n-1} g_{2}\right\|=\|y\|<K 2^{-1},
\end{gathered}
$$

and

$$
\left\|a^{n} f_{2}-a^{n} e_{3}\right\|=\left\|a\left(a^{n-1} e_{2}-a^{n-1} g_{2}\right)-a^{n} e_{3}\right\|=\left\|a^{n} e_{2}-a^{n} e_{3}\right\|<2^{-2} .
$$

Continue replacing $e_{i}$ 's by $f_{i}$ 's until at the $n$th stage we have

$$
\begin{gathered}
a^{n} f_{i}=a^{n} e_{i} \quad \text { for } i=1,2, \ldots, n, \\
\left\|a^{n-1} f_{i}-a^{n-1} f_{i+1}\right\|<K 2^{-i} \quad \text { for } i=1,2, \ldots,(n-1),
\end{gathered}
$$

and

$$
\left\|a^{n} f_{n}-a^{n} e_{n+1}\right\|<2^{-n} .
$$

Again we can find $y \in \mathcal{A}$ such that $a y=\left(a^{n} f_{n}-a^{n} e_{n+1}\right)$ and $\|y\|<K 2^{-n}$, with $y-\left(a^{n-1} f_{n}-a^{n-1} e_{n+1}\right)=h_{n+1} \in \mathcal{N}_{1} \subseteq \mathcal{T}(a)$. Since $\mathcal{T}(a)$ is $a$-divisible, we can find $g_{n+1} \in \mathcal{T}(a)$ such that $a^{n-1} g_{n+1}=h_{n+1}$ and, hence, $a^{n} g_{n+1}=0$. Let $f_{n+1}=e_{n+1}-g_{n+1}$ and compute

$$
\begin{gathered}
a\left(a^{n-1} f_{n+1}\right)=a^{n} e_{n+1}-a^{n} g_{n+1}=a^{n} e_{n+1} \\
\left\|a^{n-1} f_{n}-a^{n-1} f_{n+1}\right\|=\left\|a^{n-1} f_{n}-a^{n-1} e_{n+1}+a^{n-1} g_{n+1}\right\|=\|y\|<K 2^{-n}
\end{gathered}
$$

and

$$
\begin{gathered}
\left\|a^{n} f_{n+1}-a^{n} e_{n+2}\right\|=\left\|a\left(a^{n-1} e_{n+1}-a^{n-1} g_{n+1}\right)-a^{n} e_{n+2}\right\| \\
=\left\|a^{n} e_{n+1}-a^{n} e_{n+2}\right\|<2^{-(n+1)} .
\end{gathered}
$$

Induction now establishes the lemma.

Corollary 1.9. Let $\mathcal{A}$ be a commutative Banach algebra. Let $a \in \mathcal{A}$ satisfy a $\mathcal{A}=$ $\overline{a \mathcal{A}}$ and $\mathcal{T}(a) \subseteq a \mathcal{A}$. Then all ranges are closed $\left(a^{n} \mathcal{A}=\overline{a^{n} \mathcal{A}}\right.$ for all $\left.n \in \mathbf{N}\right)$, the ideal consisting of the elements of infinite height $\bigcap_{n=1}^{\infty} a^{n} \mathcal{A}$ is closed, and $\rho_{\sigma}(a)>0$. 
Proof. Let $n \in \mathbf{N}$ and $y \in \overline{a^{n} \mathcal{A}}$. We can certainly find a sequence $\left\{a^{n} e_{i}\right\}_{i=1}^{\infty}$ converging to $y$ satisfying $\left\|a^{n} e_{i}-a^{n} e_{i+1}\right\|<2^{-i}$ for all $i \in \mathbf{N}$. Applying Lemma 1.8 $n$ times gives us a sequence $\left\{g_{i}\right\}_{i=1}^{\infty}$ satisfying $a^{n} g_{i}=a^{n} e_{i}$ and $\left\|g_{i}-g_{i+1}\right\|<K^{n} 2^{-i}$ for all $i \in \mathbf{N}$. Since $\lim _{i \rightarrow \infty} g_{i}=g$ for some $g \in \mathcal{A}$ we must have $a^{n} g=y$. Therefore $a^{n} \mathcal{A}=\overline{a^{n} \mathcal{A}}$. Since $n$ was arbitrary, this shows that all ranges are closed. Therefore,

$$
\bigcap_{n=1}^{\infty} a^{n} \mathcal{A}=\bigcap_{n=1}^{\infty} \overline{a^{n} \mathcal{A}}=\overline{\bigcap_{n=1}^{\infty} a^{n} \mathcal{A}}
$$

and, consequently, Lemma 1.7 shows that

$$
a\left(\bigcap_{n=1}^{\infty} a^{n} \mathcal{A}\right)=\overline{\bigcap_{n=1}^{\infty} a^{n} \mathcal{A} .}
$$

If $a$ were quasi-nilpotent, we would also have

$$
\left.(\lambda-a) \overline{\left(\bigcap_{n=1}^{\infty} a^{n} \mathcal{A}\right.}\right)=\overline{\bigcap_{n=1}^{\infty} a^{n} \mathcal{A}}
$$

for all $\lambda \neq 0$ and the ideal consisting of the elements of infinite height would be $a$-divisible. Since it contains the torsion elements $\mathcal{T}(a)$ and since a quasi-nilpotent operator with closed range cannot be injective, the torsion subspace cannot be trivial (i.e., $\mathcal{T}(a) \neq\{0\}$ ). But a closed divisible subspace in a Banach space must be trivial since if $\lambda$ is in the boundary of the spectrum $\sigma(a)$ of $a$, then $(\lambda-a)$ cannot be surjective. This contradiction shows that $a$ is not quasi-nilpotent and $\rho_{\sigma}(a)>0$, finishing the proof of the corollary.

We now have a number of related technical definitions which have been used before (see [Allan1 and [Allan2, Introduction and Section 2]) and which will be important in the subsequent sections.

Definition 1.10. Let $a$ be an element in a Fréchet algebra $\mathcal{A}$.

(i) We say that $a$ has finite closed descent if there exists $n \in \mathbf{N}$ such that $a^{n} \in \overline{a^{n+1} \mathcal{A}^{\sharp}}$.

(ii) We say that $a$ has locally finite closed descent if for every $N \in \mathbf{N}$ there exists $n \in \mathbf{N}$ such that $a^{n} \in{\overline{a^{n+1} \mathcal{A}^{\sharp}}}^{N}$ (the closure is taken in the $N$ th seminorm). Note that if $\mathcal{A}$ is not a Banach space, $n$ in general will depend on $N$.

We remark that if Definition 1.10(ii) holds, it is easily checked, as a consequence of the submultiplicative property of the seminorms, that

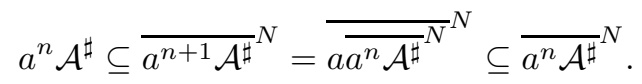

Taking closures of the above chain of containments with respect to the $N$ th seminorm shows that multiplication by $a$ on ${\overline{a^{n} \mathcal{A}^{\sharp}}}^{N}$ has $\|\cdot\|_{N}$-dense range. It is, then a routine induction to show that for every $p \in \mathbf{N}$, multiplication by $a^{p}$ on ${\overline{a^{n} \mathcal{A}^{\sharp}}}^{N}$ has $\|\cdot\|_{N}$-dense range such that

$$
{\overline{a^{n+p} \mathcal{A}^{\sharp}}}^{N}={\overline{a^{n} \mathcal{A}^{\sharp}}}^{N} \text { and } a^{n+p} \in{\overline{a^{n+p+1} \mathcal{A}^{\sharp}}}^{N} \text {. }
$$

For the next definition we stress that we are not assuming that $\mathcal{A}$ is an integral domain. 
Definition 1.11. Let $\mathcal{A}$ be a commutative Fréchet algebra. We say that an element $a \in \mathcal{A}$ divides $b \in \mathcal{A}$ (and we write $a \mid b$ ) if there exists $c \in \mathcal{A}^{\sharp}$ (note the sharp) such that $b=a c$. We say that an element $a \in \mathcal{A}$ is prime if whenever $a \mid b c$, then either $a \mid b$ or $a \mid c$. We say that an element $a \in \mathcal{A}$ is semiprime if whenever $a \mid b^{2}$, then $a \mid b$.

It is a consequence of Read1 that every semiprime element in a commutative Banach algebra has closed range. With regard to formal power series, it is too much to ask that the generator be prime (unless we are in the very special situation of the coefficient algebra being an integral domain). We will therefore work with the less stringent requirement of being weakly almost prime. The definition is unfortunately less direct than one might like. Although the two concepts can easily differ in the context of Fréchet algebras, it is an open question whether or not there exists a weakly almost prime element of a commutative radical Banach algebra that is not prime.

Definition 1.12. Let $q \in \mathcal{A}, k \in \mathbf{N}$ with $k \geq 2$ and $\left\{x_{1}, x_{2}, \ldots, x_{k}\right\} \subseteq \mathcal{A}$. We say that $\left\{x_{1}, x_{2}, \ldots, x_{k}\right\}$ satisfies subdivisibility with respect to $q$ if

$$
\begin{gathered}
q \mid x_{r} x_{s} \text { for } r<s \\
q^{2} \mid x_{r} x_{s} x_{t} \text { for } r<s<t \\
\ldots \\
q^{k-1} \mid x_{1} x_{2} \ldots x_{k} .
\end{gathered}
$$

We say that $q$ is almost $k$-prime if whenever $\left\{x_{1}, x_{2}, \ldots, x_{k}\right\}$ satisfies subdivisibility with respect to $q$, then there exists $\left\{u_{1}, u_{2}, \ldots, u_{k}\right\} \subseteq \mathcal{A}^{\sharp}$ such that

$$
\prod_{j=1}^{k}\left(x_{j}-q u_{j}\right)=0 .
$$

We say that $q$ is weakly almost $k$-prime if whenever $\left\{x_{1}, x_{2}, \ldots, x_{k}\right\}$ satisfies subdivisibility with respect to $q$, then there exists $\left\{u_{1}, u_{2}, \ldots, u_{k}\right\} \subseteq \mathcal{A}^{\sharp}$ such that

$$
\prod_{j=1}^{k}\left(x_{j}-q u_{j}\right) \in q^{k} \mathcal{A}^{\sharp} .
$$

Definition 1.13. Let $p$ be an element of $\mathcal{A}$. We say that $p$ is almost prime if $p^{k}$ is almost $(k+1)$-prime for all $k \in \mathbf{N}$. We say that $p$ is weakly almost prime if $p^{k}$ is weakly almost $(k+1)$-prime for all $k \in \mathbf{N}$.

\section{§2. Local Power Series Quotients}

We are interested in knowing when a commutative Fréchet algebra $\mathcal{A}^{\sharp}$ has a (unital) algebra homomorphism $\theta$ that is onto an algebra of formal series with coefficients in some commutative unital complex algebra $\mathcal{A}_{0}$. We will use the notation

$$
\mathcal{A}_{0}[[X]] \equiv\left\{\sum_{i=0}^{\infty} a_{i} X^{i} \mid a_{i} \in \mathcal{A}_{0}\right\}
$$

for such an algebra of formal series. If the kernel of $\theta$ is the ideal $\mathcal{J}$, then $\theta$ factors through $\mathcal{J}$ as an isomorphism $\theta \cong$ from $\mathcal{A}^{\sharp} / \mathcal{J}$ onto $\mathcal{A}_{0}[[X]]$. We do not, at this point, assume any topology on $\mathcal{A}_{0}$. Unfortunately, if we put no restrictions on $\mathcal{J}$, there may be some very ill-behaved isomorphisms (see [Dales-McClure, Theorem 2.3]) even in the case where $\mathcal{A}_{0} \cong \mathbb{C}$. 
If there is a unital algebra homomorphism $\theta$ from $\mathcal{A}^{\sharp}$ onto $\mathcal{A}_{0}[[X]]$ with the kernel of $\theta$ equal to $\mathcal{J}$ and associated isomorphism $\theta \cong$ from $\mathcal{A}^{\sharp} / \mathcal{J}$ onto $\mathcal{A}_{0}[[X]]$, then some element $x$ must be mapped to the indeterminate $X$, and since $X$ is not invertible in $\mathcal{A}_{0}[[X]]$, it must be the case that $x$ is not invertible in $\mathcal{A}^{\sharp}$. If $\theta(x)=X$, then, considering the ideal consisting of the elements of infinite height (with respect to $x$ ), it is clear that $\bigcap_{i=0}^{\infty} x^{n} \mathcal{A}$ must be contained in the kernel $\mathcal{J}$ since $X$ has no elements of infinite height in $\mathcal{A}_{0}[[X]]$. Since $\bigcap_{i=0}^{\infty} x^{n} \mathcal{A}$ has a (formally) stronger Fréchet topology, it is therefore natural to investigate the special case where the kernel of $\theta$ is precisely the ideal consisting of the elements of infinite height (with respect to $x$ ). We call this a formal power series quotient based at $x$ and refer to $x$ as the generating element.

Definition 2.1. Let $x \in \mathcal{A}$ and suppose that the ideal generated by $x$, namely $x \mathcal{A}^{\sharp}$, is a nonzero, proper ideal of $\mathcal{A}$ (this excludes trivial cases). We say that the commutative Fréchet algebra $\mathcal{A}$ has a formal power series quotient based at $x$ if there exists a commutative unital complex algebra $\mathcal{A}_{0}$ and a unital algebra homomorphism $\theta$ onto $\mathcal{A}_{0}[[X]]$ such that the kernel of $\theta$ is precisely the ideal consisting of the elements of infinite height; that is,

$$
\theta \cong:\left(\mathcal{A}^{\sharp} / \bigcap_{n=1}^{\infty} x^{n} \mathcal{A}\right) \cong \mathcal{A}_{0}[[X]]
$$

and $\theta(x)=X$. We say that a commutative Fréchet algebra $\mathcal{A}$ has a local power series quotient if it has a formal power series quotient based at $x$ for some $x \in \mathcal{A}$ for which the ideal generated by $x$ is nonzero and proper.

We remark that Definition 2.1 generalizes to non-commutative Fréchet algebras by replacing the kernel of $\theta$ above, $\bigcap_{n=1}^{\infty} x^{n} \mathcal{A}$, by $\bigcap_{n=1}^{\infty}\left(\mathcal{C}+x^{n} \mathcal{A}\right)$ where $\mathcal{C}$ is the ideal generated by the commutators $\{x y-y x \mid x, y \in \mathcal{A}\}$. However, this has the unfortunate consequence that the kernel of $\theta$ is no longer an intersection of range spaces.

We require several lemmas. The first lemma, a somewhat more general MittagLeffler theorem (see [Sinclair]) is proved by essentially the same method as in Thomas1, Lemma 1.1d], although the maps here are not assumed to commute.

Lemma 2.2. Let $\left\{X_{n}\right\}_{n=1}^{\infty}$ be closed subspaces of a Fréchet space $\left(X,\|\cdot\|_{n}\right)$ (with $\left.\|\cdot\|_{n} \leq\|\cdot\|_{n+1}\right)$ and let $\left\{\varphi_{n}: X_{n+1} \rightarrow X_{n}\right\}_{n=1}^{\infty}$ be a sequence of continuous functions satisfying:

(i) There exists a sequence of constants $\left\{C_{i}\right\}_{i=1}^{\infty}$ such that for all $a, b \in X_{n+1}$ and nonnegative integers $p$ we have

$$
\left\|\varphi_{n}(a)-\varphi_{n}(b)\right\|_{n+p} \leq C_{n+p}\|a-b\|_{n+p}
$$

for $n=1,2, \ldots$.

(ii) $\overline{\varphi_{n}\left(X_{n+1}\right)}{ }^{n} \supseteq X_{n}$ for $n=1,2, \ldots$.

Then the inverse, or projective, limit $P=\left\{\left(y_{n}\right)_{n=1}^{\infty} \mid \varphi_{n} y_{n+1}=y_{n}\right.$ for $\left.n=1,2, \ldots\right\}$ is nonempty and ${\overline{\pi_{n}(P)}}^{n} \supseteq X_{n}$ (where $\pi_{n}$ is the nth coordinate projection) for $n=1,2, \ldots$

Proof. Without loss of generality, we may assume that $C_{i} \geq 1$ for all $i \in \mathbf{N}$. Fix $n \in \mathbf{N}$ and $\epsilon>0$. Let $x_{n}$ be any element of $X_{n}$. Choose $x_{n+1} \in X_{n+1}$ such that $\left\|\varphi_{n} x_{n+1}-x_{n}\right\|_{n}<\epsilon /\left(2^{n} C_{n}\right)$. Continue inductively choosing $x_{n+p+1} \in X_{n+p+1}$ 
such that

$$
\left\|\varphi_{n+p} x_{n+p+1}-x_{n+p}\right\|_{n+p}<\epsilon /\left(2^{n+p+1} C_{n+p}^{p}\right)
$$

for $p=1,2, \ldots$ Given any nonnegative integer $j$, observe that

$$
\begin{gathered}
\left\|\varphi_{n+j} x_{n+j+1}-x_{n+j}\right\|_{n+j} \\
+\sum_{p=j+1}^{\infty}\left\|\varphi_{n+j} \varphi_{n+j+1} \ldots \varphi_{n+p} x_{n+p+1}-\varphi_{n+j} \varphi_{n+j+1} \ldots \varphi_{n+p-1} x_{n+p}\right\|_{n+p} \\
\leq\left\|\varphi_{n+j} x_{n+j+1}-x_{n+j}\right\|_{n+j} \\
+\sum_{p=j+1}^{\infty} C_{n+p}\left\|\varphi_{n+j+1} \ldots \varphi_{n+p} x_{n+p+1}-\varphi_{n+j+1} \ldots \varphi_{n+p-1} x_{n+p}\right\|_{n+p} \\
\ldots \\
\leq \sum_{p=j}^{\infty} C_{n+p}^{p-j}\left\|\varphi_{n+p} x_{n+p+1}-x_{n+p}\right\|_{n+p} \\
\leq \sum_{p=j}^{\infty} C_{n+p}^{p-j} \epsilon /\left(2^{n+p+1} C_{n+p}^{p}\right) \leq \epsilon /\left(2^{n+j}\right) .
\end{gathered}
$$

This shows that the sequence $\left\{\varphi_{n+j} \ldots \varphi_{n+p} x_{n+p+1}\right\}_{p=j}^{\infty}$ is Cauchy (in $p$ ) in the Fréchet topology of $X$ (and $X_{n+j}$, which is closed). Consequently, there exists $y_{n+j} \in X_{n+j}$ such that

$$
\varphi_{n+j} \ldots \varphi_{n+p} x_{n+p+1} \rightarrow y_{n+j} \text { as } p \rightarrow \infty
$$

for $j=0,1,2, \ldots$ But if we also define $y_{n-1}=\varphi_{n-1} y_{n}, y_{n-2}=\varphi_{n-2} \varphi_{n-1} y_{n}, \ldots$ $y_{1}=\varphi_{1} \varphi_{2} \ldots \varphi_{n-1} y_{n}$, then it is routine to show that $\left(y_{i}\right)_{i=1}^{\infty} \in P$ and $\pi_{n}\left(y_{i}\right)_{i=1}^{\infty}=$ $y_{n}$.

Finally, we can compute the $n$th seminorm distance from $\pi_{n}\left(y_{i}\right)_{i=1}^{\infty}$ and $x_{n}$ as

$$
\begin{gathered}
\left\|y_{n}-x_{n}\right\|_{n} \leq \lim _{m \rightarrow \infty}\left\|\varphi_{n} \ldots \varphi_{n+m} x_{n+m+1}-x_{n}\right\|_{n} \\
\leq \lim _{m \rightarrow \infty}\left\|\sum_{p=0}^{m} \varphi_{n} \ldots \varphi_{n+p} x_{n+p+1}-\varphi_{n} \ldots \varphi_{n+p-1} x_{n+p}\right\|_{n+p} \\
\leq \sum_{p=0}^{\infty}\left\|\varphi_{n} \ldots \varphi_{n+p} x_{n+p+1}-\varphi_{n} \ldots \varphi_{n+p-1} x_{n+p}\right\|_{n+p} \\
\leq \sum_{p=0}^{\infty} \epsilon /\left(2^{n+p+1}\right) \leq \epsilon .
\end{gathered}
$$

Since $\epsilon>0$ was arbitrary, this shows that the closure in the $n$th seminorm ${\overline{\pi_{n}(P)}}^{n}$ contains $X_{n}$.

Lemma 2.3. Let $x$ be an element of a commutative Fréchet algebra $\mathcal{A}$, let $\dot{x}$ denote the coset containing $x$ in the quotient Fréchet algebra $\mathcal{A}^{\sharp} / \overline{\bigcap_{m=1}^{\infty} x^{m} \mathcal{A}}$. Then either there exists $n \in \mathbf{N}$ such that

$$
x^{k} \notin{\overline{x^{k+1} \mathcal{A}^{\sharp}}}^{n}
$$

for all $k \in \mathbf{N}$ (i.e., $x$ does not have locally finite closed descent) or $\dot{x}$ is locally nilpotent. 
Proof. Suppose that the first alternative fails and that no such $n$ exists. Then for any $n \in \mathbf{N}$ we can find $k \in \mathbf{N}$ such that

$$
x^{k} \in{\overline{x^{k+1} \mathcal{A}^{\sharp}}}^{n}={\overline{x\left(x^{k} \mathcal{A}^{\sharp}\right)}}^{n} .
$$

Our remark after Definition 1.10 shows that

$$
{\overline{x^{k+p} \mathcal{A}^{\sharp}}}^{n}={\overline{x^{p}\left(x^{k} \mathcal{A}^{\sharp}\right.}}^{n}={\overline{x \overline{\left.x^{k+p-1} \mathcal{A}^{\sharp}\right)}}}^{n}={\overline{x{\overline{\left(x^{k} \mathcal{A}^{\sharp}\right.}}^{n}}}^{n}={\overline{x^{k} \mathcal{A}^{\sharp}}}^{n}
$$

for $p=1,2, \ldots$ It is therefore possible to choose an increasing sequence $\left\{k_{n}\right\}_{n=1}^{\infty}$ such that

$$
{\overline{x\left(x^{k_{n+1}} \mathcal{A}^{\sharp}\right)}}^{n}={\overline{x^{k_{n}} \mathcal{A}^{\sharp}}}^{n}
$$

for $n=1,2, \ldots$. Therefore, for each $n \in \mathbf{N}$, let

$$
X_{n}={\overline{x^{k_{n}} \mathcal{A}^{\sharp}}}^{n},
$$

let $\varphi_{n}$ be multiplication by $x$ (which is a continuous function from $X_{n+1}$ to $X_{n}$ ) and let $C_{n}=\|x\|_{n}$. Note that the hypotheses of Lemma 2.2 are satisfied, namely:

(i) For all $n, p \in \mathbf{N}$ the difference in the $(n+p)$ th seminorms has the correct upper bound

$$
\left\|\varphi_{n}(a)-\varphi_{n}(b)\right\|_{n+p}=\|x(a-b)\|_{n+p} \leq\|x\|_{n+p}\|a-b\|_{n+p}=C_{n+p}\|a-b\|_{n+p} .
$$

(ii) For all $n \in \mathbf{N}$, the maps have dense range

$$
{\overline{\varphi_{n}\left(X_{n+1}\right)}}^{n}={\overline{x{\overline{\left(x^{k_{n+1}} \mathcal{A}^{\sharp}\right.}}^{n+1}}}^{n}={\overline{x\left(x^{k_{n+1}} \mathcal{A}^{\sharp}\right)}}^{n}={\overline{x^{k_{n}} \mathcal{A}^{\sharp}}}^{n}=X_{n} .
$$

Lemma 2.2 can therefore be applied. In this case, it is easily seen that the image of any canonical projection of the projective limit is simply the largest subspace $H \subseteq \mathcal{A}^{\sharp}$ satisfying $x H=H$ (i.e., the subspace is divisible by the single operation of multiplication by $x$ ), such that

$$
\bar{H}^{n} \supseteq{\overline{x^{k_{n}} \mathcal{A}^{\sharp}}}^{n} \supseteq x^{k_{n}} \mathcal{A}^{\sharp}
$$

for $n=1,2, \ldots$. Since we must have $H \subseteq\left(\bigcap_{m=1}^{\infty} x^{m} \mathcal{A}\right)$, it follows that

$$
x^{k_{n}} \in x^{k_{n}} \mathcal{A}^{\sharp} \subseteq \bar{H}^{n} \subseteq{\overline{\bigcap_{m=1}^{\infty} x^{m} \mathcal{A}}}^{n}
$$

for $n=1,2, \ldots$ This shows that $\dot{x}$ is locally nilpotent and completes the proof.

Next we observe that the inductive construction (of what we called a recalcitrant system) in [Thomas2] of sections $3.10 \mathrm{a}-3.10 \mathrm{~d}$ can be reworded in our setting to equivalently read:

Lemma 2.4 ([Thomas2]). Let $s$ be a non-nilpotent element of a commutative radical Banach algebra $\mathcal{R}^{\sharp}$ with identity adjoined (so that $\mathcal{R}^{\sharp} \cong \mathbb{C} \cdot 1 \oplus \mathcal{R}$ and $\mathcal{R}=$ $\left.\operatorname{rad}\left(\mathcal{R}^{\sharp}\right)\right)$. Let $\left\{e_{\alpha}\right\}_{\alpha \in \mathbf{N}}$ be a sequence of elements in $\mathcal{R}$ such that $\lim _{\alpha \rightarrow \infty} e_{\alpha} s=s$. Fix a positive integer $k \in \mathbf{N}$ and choose $k$ fixed sequences $\left\{\left\{\lambda_{i j}\right\}_{i=1}^{k}\right\}_{j=1}^{\infty}$ satisfying

$$
\lim _{j \rightarrow \infty}\left|\lambda_{i 1} \lambda_{i 2} \ldots \lambda_{i j}\right|=+\infty
$$

for $i=1,2, \ldots, k$. Order the set of double indices $\left\{\{i j\}_{i=1}^{k}\right\}_{j=1}^{\infty}$ with the following lexicographical order: $i_{1} j_{1}<i_{2} j_{2}$ if either $j_{1}<j_{2}$ or $j_{1}=j_{2}$ and $i_{1}<i_{2}$. Once $\alpha_{i j}$ has been chosen, the element $\left(\lambda_{i j}+\left(1-\lambda_{i j}\right) e_{\alpha_{i j}}\right)$ will be invertible in $\mathcal{R}^{\sharp}$. Then, as 
long as the values $\left\{\left\{\alpha_{i j}\right\}_{i=1}^{k}\right\}_{j=1}^{\infty}$ are chosen to increase sufficiently rapidly as the $\left\{\{i j\}_{i=1}^{k}\right\}_{j=1}^{\infty}$ increase in the lexicographical order, the elements

$$
s_{i} \equiv s \prod_{j=1}^{\infty}\left(\lambda_{i j}+\left(1-\lambda_{i j}\right) e_{\alpha_{i j}}\right)
$$

converge in $\mathcal{R}$ for $i=1,2, \ldots, k$, satisfy subdivisibility with respect to $s$, and have the property

$$
\sup _{n} \frac{\left\|\prod_{i=1}^{k}\left(s_{i}-s v_{i}\right)^{n}\right\|^{1 / n}}{\left\|s^{k n}\right\|^{1 / n}}=+\infty
$$

for all choices of $\left\{v_{1}, v_{2}, \ldots, v_{k}\right\} \subseteq \mathcal{R}^{\sharp}$.

We note that the requirement in the above lemma that the $\left\{e_{\alpha}\right\}_{\alpha \in \mathbf{N}}$ be in the radical $\mathcal{R}$ (and not simply in $\mathcal{R}^{\sharp}$ ) is essential for the construction given in [Thomas2]. We wish to extend this result to radical Fréchet algebras, but the more stringent requirement that the element be nonlocally nilpotent is necessary.

Corollary 2.5. Let $\mathcal{R}^{\sharp}$ be a commutative radical Fréchet algebra with identity adjoined (so that $\mathcal{R}^{\sharp} \cong \mathbb{C} \cdot 1 \oplus \mathcal{R}$ and $\mathcal{R}=\operatorname{rad}\left(\mathcal{R}^{\sharp}\right)$ ) and let $x$ be a nonlocally nilpotent element of $\mathcal{R}$ satisfying

$$
x^{m_{0}} \in \overline{x^{m_{0}} \mathcal{R}}
$$

for some $m_{0} \in \mathbf{N}$. Then there exists $n_{0} \in \mathbf{N}$, and elements $\left\{s_{1}, s_{2}, \ldots, s_{m_{0}+1}\right\} \subseteq \mathcal{R}$ such that $\left\{s_{1}, s_{2}, \ldots, s_{m_{0}+1}\right\}$ satisfy subdivisibility with respect to $x^{m_{0}}$ and

$$
\sup _{n} \frac{\left\|\prod_{i=1}^{m_{0}+1}\left(s_{i}-x^{m_{0}} u_{i}\right)^{n}\right\|_{n_{0}}^{1 / n}}{\left\|x^{m_{0}\left(m_{0}+1\right) n}\right\|_{n_{0}}^{1 / n}}=+\infty
$$

for all choices of $\left\{u_{1}, u_{2}, \ldots, u_{m_{0}+1}\right\} \subseteq \mathcal{R}^{\sharp}$. Consequently, $x$ is not weakly almost prime.

Proof. Since $x$ is nonlocally nilpotent, we can choose $n_{0} \in \mathbf{N}$ such that $\left\|x^{n}\right\|_{n_{0}} \neq 0$ for all $n \in \mathbf{N}$. Let $k=m_{0}+1$ and choose $k$ fixed sequences $\left\{\left\{\lambda_{i j}\right\}_{i=1}^{k}\right\}_{j=1}^{\infty}$ satisfying

$$
\lim _{j \rightarrow \infty}\left|\lambda_{i 1} \lambda_{i 2} \ldots \lambda_{i j}\right|=+\infty
$$

for $i=1,2, \ldots, k$. We have, as a consequence of the Arens-Michael isomorphism, a continuous homomorphism $\pi_{n_{0}}$ (with dense range) from $\mathcal{R}^{\sharp}$ into $\mathcal{B}_{n_{0}}$ with kernel $\mathcal{I}_{n_{0}}$. It is easy to verify that $\mathcal{B}_{n_{0}}$ is also a radical algebra with identity adjoined since for all $r \in \mathcal{R}$

$$
\{0\}=\sigma(r)=\bigcup_{n=1}^{\infty} \sigma_{n}(r)
$$

as noted in the discussion of the Arens-Michael isomorphism in Section 1. But the spectrum is nonempty in each $\mathcal{B}_{n}$ and so $\sigma_{n_{0}}(r)=\sigma\left(\pi_{n_{0}}(r)\right)=\{0\}$ for all $r \in \mathcal{R}$. This, together with the fact that $\pi_{n_{0}}$ has dense range, shows that $\mathcal{B}_{n_{0}}$ is a commutative radical Banach algebra with identity adjoined. Let $s=\left(\pi_{n_{0}}(x)\right)^{m_{0}}$. Since $x^{m_{0}} \in \overline{x^{m_{0}} \mathcal{R}}$, we can find a sequence $\left\{f_{\alpha}\right\}_{\alpha \in \mathbf{N}}$ in $\mathcal{R}$ such that $f_{\alpha} x^{m_{0}} \rightarrow x^{m_{0}}$ in the Fréchet topology of $\mathcal{R}$. If we let $e_{\alpha}=\pi_{n_{0}}\left(f_{\alpha}\right)$ for each $\alpha \in \mathbf{N}$, it will follow that $\lim _{\alpha \rightarrow \infty} e_{\alpha} s=s$ in the norm topology of the commutative radical Banach algebra 
$\mathcal{B}_{n_{0}}$ with identity adjoined. If $\left\{\left\{\alpha_{i j}\right\}_{i=1}^{k}\right\}_{j=1}^{\infty}$ are chosen to increase sufficiently rapidly as the $\left\{\{i j\}_{i=1}^{k}\right\}_{j=1}^{\infty}$ increase in the lexicographical order, then the elements

$$
s_{i} \equiv x^{m_{0}} \prod_{j=1}^{\infty}\left(\lambda_{i j}+\left(1-\lambda_{i j}\right) f_{\alpha_{i j}}\right)
$$

will converge in the Fréchet topology of $\mathcal{R}$ and satisfy subdivisibility with respect to $x^{m_{0}}$, the conditions of Lemma 2.4 will be met, and the elements

$$
\pi_{n_{0}}\left(s_{i}\right) \equiv s \prod_{j=1}^{\infty}\left(\lambda_{i j}+\left(1-\lambda_{i j}\right) e_{\alpha_{i j}}\right)
$$

will converge in the Banach algebra topology of $\mathcal{B}_{n_{0}}$, and

$$
\sup _{n} \frac{\left\|\prod_{i=1}^{k}\left(\pi_{n_{0}}\left(s_{i}\right)-s v_{i}\right)^{n}\right\|^{1 / n}}{\left\|s^{k n}\right\|^{1 / n}}=+\infty
$$

for all choices of $\left\{v_{1}, v_{2}, \ldots, v_{k}\right\} \subseteq \mathcal{B}_{n_{0}}$. Remembering that for $u \in \mathcal{R}^{\sharp},\|u\|_{n_{0}}=$ $\left\|\pi_{n_{0}}(u)\right\|$, we conclude that given any $k=m_{0}+1$ choices $\left\{u_{1}, u_{2}, \ldots, u_{k}\right\} \subseteq \mathcal{R}^{\sharp}$, we can apply the above to $v_{i}=\pi_{n_{0}}\left(u_{i}\right)$ for $i=1,2, \ldots, k$ and obtain

$$
\sup _{n} \frac{\left\|\prod_{i=1}^{m_{0}+1}\left(s_{i}-x^{m_{0}} u_{i}\right)^{n}\right\|_{n_{0}}^{1 / n}}{\left\|x^{m_{0}\left(m_{0}+1\right) n}\right\|_{n_{0}}^{1 / n}}=+\infty
$$

Suppose now that $x$ is weakly almost prime. Then $x^{m_{0}}$ would be weakly almost $\left(m_{0}+1\right)$-prime. However, the set $\left\{s_{1}, s_{2}, \ldots, s_{m_{0}+1}\right\}$ satisfies subdivisibility with respect to $x^{m_{0}}$ and there would have to exist $\left\{u_{1}, u_{2}, \ldots, u_{m_{0}+1}\right\} \subseteq \mathcal{R}^{\sharp}$ and $u \in \mathcal{R}^{\sharp}$ such that

$$
\prod_{i=1}^{m_{0}+1}\left(s_{i}-x^{m_{0}} u_{i}\right)=x^{m_{0}\left(m_{0}+1\right)} u .
$$

But $\sup _{n}\left\|u^{n}\right\|_{n_{0}}^{1 / n}=\rho_{\sigma}\left(\pi_{n_{0}}(u)\right)$ is bounded in the Banach algebra $\mathcal{B}_{n_{0}}$ contradicting

$$
\sup _{n} \frac{\left\|\prod_{i=1}^{m_{0}+1}\left(s_{i}-x^{m_{0}} u_{i}\right)^{n}\right\|_{n_{0}}^{1 / n}}{\left\|x^{m_{0}\left(m_{0}+1\right) n}\right\|_{n_{0}}^{1 / n}}=+\infty
$$

and showing that such a $u \in \mathcal{R}^{\sharp}$ cannot exist. Therefore, $x$ cannot be weakly almost prime.

We now have our result which establishes the main necessary conditions for having a local power series quotient. We note that the seventh conclusion (vii) is the most important since it shows that either $x$ has finite closed descent and $\mathcal{A}^{\sharp}$ has nontrivial representation theory, or $x$ does not have finite closed descent, $\mathcal{A}^{\sharp}$ is not a Banach algebra, and $\dot{x}$ must be locally nilpotent (but non-nilpotent) in the quotient Fréchet algebra $\mathcal{A}^{\sharp} / \overline{\bigcap_{m=1}^{\infty} x^{m} \mathcal{A}}$.

Proposition 2.6. Suppose that the commutative Fréchet algebra $\mathcal{A}$ has a formal power series quotient based at $x$, let $\theta$ denote the surjective homomorphism for which $\theta(x)=X$, let $\theta \cong$ denote the associated isomorphism

$$
\theta \cong:\left(\mathcal{A}^{\sharp} / \bigcap_{n=1}^{\infty} x^{n} \mathcal{A}\right) \cong \mathcal{A}_{0}[[X]],
$$


and let $\dot{x}$ be the coset containing $x$ in the quotient Fréchet algebra $\mathcal{A}^{\sharp} / \overline{\bigcap_{m=1}^{\infty} x^{m} \mathcal{A}}$. Then the following conditions hold:

(i) $x$ is not nilpotent;

(ii) the torsion elements $\mathcal{T}(x)$ with respect to $x$ are contained in $\bigcap_{n=1}^{\infty} x^{n} \mathcal{A}$ and hence $x\left(\bigcap_{n=1}^{\infty} x^{n} \mathcal{A}\right)=\left(\bigcap_{n=1}^{\infty} x^{n} \mathcal{A}\right)$;

(iii) (algebraically) $\mathcal{A}_{0} \cong \mathcal{A}^{\sharp} / x \mathcal{A}^{\sharp}$;

(iv) if $\mathcal{A}$ contains an idempotent $e$ different from the identity, and if $e$ is not contained in the kernel $\left(\bigcap_{n=1}^{\infty} x^{n} \mathcal{A}\right)$ of $\theta$, then $b_{0}=\theta(e)$ is a nonzero idem-

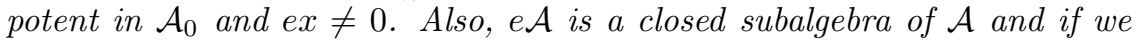
define $\theta_{e}(a)=\theta(e a)$, we have the associated isomorphism:

$$
\theta_{e} \cong:\left(e \mathcal{A} / \bigcap_{n=1}^{\infty}(e x)^{n} e \mathcal{A}\right) \cong\left(b_{0} \mathcal{A}_{0}\right)[[X]]
$$

so that $e \mathcal{A}$ has a formal power series quotient based at (ex);

(v) either $\mathcal{A}_{0}$ contains nonzero idempotents other than the identity element or whenever $x=u v$ for $u, v \in \mathcal{A}^{\sharp}$ one of $\{u, v\}$ is invertible modulo the kernel $\left(\bigcap_{n=1}^{\infty} x^{n} \mathcal{A}\right)$ of $\theta$ and, consequently, $x$ is irreducible;

(vi) $y$ is divisible by $x^{n}$ if and only if $\theta(y)=\sum_{k=0}^{\infty} y_{k} X^{k}$ satisfies $y_{0}=y_{1}=$ $\ldots=y_{n-1}=0$, and, consequently, $x$ is weakly almost prime;

(vii) either $x$ has finite closed descent and $\mathcal{A}^{\sharp}$ is not a commutative radical Fréchet algebra with identity adjoined or $\mathcal{A}$ is not a Banach algebra, $x$ does not have finite closed descent, $\sigma(\dot{x})=\{0\}$, and $\dot{x}$ is locally nilpotent (but non-nilpotent).

Proof. Since $\theta(x)=X$ and since $X$ is non-nilpotent, $x$ is non-nilpotent. This proves (i).

Since $\mathcal{A}_{0}[[X]]$ has no $X$-torsion, it must be the case that $\mathcal{T}(x)$ is contained in the kernel of $\theta$ which is precisely $\bigcap_{n=1}^{\infty} x^{n} \mathcal{A}$. This means that $\mathcal{T}(x) \subseteq \bigcap_{n=1}^{\infty} x^{n} \mathcal{A} \subseteq x \mathcal{A}$ and an application of Lemma 1.7 establishes (ii).

Note that $\mathcal{A}_{0} \cong \mathcal{A}_{0}[[X]] /\left(X \mathcal{A}_{0}[[X]]\right)$. Consideration of the preimages shows that

$$
\mathcal{A}^{\sharp} / x \mathcal{A}^{\sharp} \cong\left(\mathcal{A}^{\sharp} / \bigcap_{n=1}^{\infty} x^{n} \mathcal{A}\right) /\left(x \mathcal{A}^{\sharp} / \bigcap_{n=1}^{\infty} x^{n} \mathcal{A}\right) / \cong \mathcal{A}_{0}[[X]] /\left(X \mathcal{A}_{0}[[X]]\right)
$$

and establishes (iii).

Suppose that $e$ is an idempotent in $\mathcal{A}$ different from the identity. If $e \notin$ $\bigcap_{n=1}^{\infty} x^{n} \mathcal{A}$, then $\theta(e)=b_{0}+\sum_{i=1}^{\infty} b_{i} X^{i}$ is a nonzero idempotent in $\mathcal{A}_{0}[[X]]$. It is clear that $b_{0}^{2}=b_{0}$. We claim that $b_{i}=0$ for all $i \geq 1$. First note that $b_{1}=2 b_{0} b_{1}$ since $\theta(e)^{2}=\theta(e)$. But, multiplying both sides by $b_{0}$ yields $b_{0} b_{1}=2 b_{0} b_{1}$ which forces $b_{0} b_{1}=0$ and, in consequence, $b_{1}=0$. Suppose that we have shown that $b_{1}=b_{2}=\ldots=b_{n-1}=0$. Then $\theta(e)=b_{0}+\sum_{i=n}^{\infty} b_{i} X^{i}$ and idempotence again requires that $b_{n}=2 b_{0} b_{n}$. Multiplication by $b_{0}$ again shows that $b_{0} b_{n}=2 b_{0} b_{n}$ and $b_{n}=0$. Induction now shows that $\theta(e)=b_{0} \neq 0$, since we are assuming that $e$ is not in the kernel of $\theta$. This also shows that $e x \neq 0$, since $\theta(e x)=b_{0} X \neq 0$. Hence, $b_{0} \mathcal{A}_{0}$ is a commutative unital complex algebra with identity $b_{0}$. Correspondingly, $e \mathcal{A}$ is a subalgebra of $\mathcal{A}$ with identity $e$. It is closed, since it is the null space of the multiplication operator $M_{1-e}$ on $\mathcal{A}$ where $M_{1-e}(a)=(a-e a)$. Define $\theta_{e}$ on $e \mathcal{A}$ by $\theta_{e}(e a)=b_{0} \theta(a)$, which is just the restriction of $\theta$ to $e \mathcal{A}$. This map is clearly onto $b_{0} \mathcal{A}_{0}[[X]]$. If $e a$ is in the kernel of $\theta_{e}$, this means that $e a \in x^{n} \mathcal{A}$ for 
all $n \in \mathbf{N}$. Multiplying by $e$ we have that $e a \in e x^{n} \mathcal{A}=(e x)^{n} e \mathcal{A}$ for all $n \in \mathbf{N}$. This establishes the surjective homomorphism $\theta_{e}$ and associated isomorphism $\theta_{e} \cong$. Suppose that $e x \mathcal{A}=e \mathcal{A}$. Apply this $n$ times to obtain that $x^{n}(e \mathcal{A})=e \mathcal{A}$ for all $n \in \mathbf{N}$. But then $e=e^{2} \in e \mathcal{A} \in \bigcap_{n=1}^{\infty} x^{n} \mathcal{A}$, a contradiction to the fact that $e$ is not in the kernel of $\theta$. We remark that this is where we need $e \in \mathcal{A}$ rather than the weaker assumption $e \in \mathcal{A}^{\sharp}$. Therefore, $e \mathcal{A}$ has a formal power series quotient based at $(e x)$. This establishes (iv).

Suppose that $x=u v$ for some $u, v \in \mathcal{A}^{\sharp}$. Apply $\theta$ so that we have $X=\theta(u) \theta(v)$ and let the expansions be $\theta(u)=\sum_{i=0}^{\infty} a_{i} X^{i}$ and $\theta(v)=\sum_{i=0}^{\infty} b_{i} X^{i}$. Computation shows that $a_{0} b_{0}=0$ and $a_{0} b_{1}+a_{1} b_{0}=1$. Consequently, one of $\left\{a_{0} b_{1}, a_{1} b_{0}\right\}$ must be nonzero. Without loss of generality, assume that $a_{0} b_{1} \neq 0$. Multiply the equation $a_{0} b_{1}+a_{1} b_{0}=1$ by $a_{0} b_{1}$ and use commutativity and the fact that $a_{0} b_{0}=0$ to conclude that

$$
\left(a_{0} b_{1}\right)^{2}=a_{0}^{2} b_{1}^{2}=a_{0} b_{1} .
$$

So $e_{0}=a_{0} b_{1}$ is a nonzero idempotent, and clearly $X$ factors as $\left(e_{0}+\left(1-e_{0}\right) X\right)((1-$ $\left.\left.e_{0}\right)+e_{0} X\right)$. However, if the only nonzero idempotent of $\mathcal{A}_{0}$ is 1 , we conclude $a_{0} b_{1}=1$ so that

$$
b_{1} \theta(u)=1-\sum_{i=1}^{\infty}\left(-b_{1} a_{i}\right) X^{i}
$$

If $\lambda \neq 0$, then any element of the form $\lambda-\sum_{i=1}^{\infty} c_{i} X^{i}$ is invertible in $\mathcal{A}_{0}[[X]]$ as follows:

$$
(\lambda-Y)^{-1}=\lambda^{-1}\left(1-\left(\lambda^{-1} Y\right)\right)^{-1}=\sum_{i=0}^{\infty} \lambda^{-(i+1)} Y^{i}
$$

for any $Y \in X \mathcal{A}_{0}[[X]]$. The series converges since each $i$ th power term of the result only depends on finitely many terms of the sum. Hence, there exists $y \in \mathcal{A}^{\sharp}$ such that $u y \in\left(1+\bigcap_{n=1}^{\infty} x^{n} \mathcal{A}\right)$, so that $u$ is invertible modulo the kernel of $\theta$. In addition, multiplying the above relation by $v$ shows that $x y=u v y \in\left(v+\bigcap_{n=1}^{\infty} x^{n} \mathcal{A}\right)$; so there exists $a \in \mathcal{A}$ such that $x y=v+x a$ or $v=x(y-a)$ and, hence, $x \mid v$. This shows that $x$ is irreducible, and completes the proof of assertion (v).

Suppose that $y \in \mathcal{A}$ and $y=x^{n} z$ for some $n \in \mathbf{N}$ and $z \in \mathcal{A}^{\sharp}$. Apply the homomorphism $\theta$ and suppose that $\theta(y)=\sum_{k=1}^{\infty} y_{k} X^{k}$ with $y_{k} \in \mathcal{A}_{0}$ for $k=1,2, \ldots$ and $\theta(z)=\sum_{k=1}^{\infty} z_{k} X^{k}$ with $z_{k} \in \mathcal{A}_{0}$ for $k=1,2, \ldots$. Since $y=x^{n} z$, it must be the case that $\sum_{k=1}^{\infty} y_{k} X^{k}=\sum_{k=n}^{\infty} z_{k-n} X^{k}$ in $\mathcal{A}_{0}[[X]]$. This can only happen if $y_{0}=y_{1}=y_{2}=\ldots=y_{n-1}=0$. Conversely, if the series for $\theta(y)$ is of the form $\sum_{k=n}^{\infty} y_{k} X^{k}$, then it is clear that $y \in x^{n} \mathcal{A}^{\sharp}$ since $\theta$ is onto and its kernel is precisely $\bigcap_{m=1}^{\infty} x^{m} \mathcal{A}$ which contains $x^{n} \mathcal{A}^{\sharp}$. Let $m_{0}$ be any positive integer. We need to show that $x^{m_{0}}$ is weakly almost $\left(m_{0}+1\right)$-prime. But the demonstration of this fact can be done in exactly the same manner as the proof of [Thomas2, Theorem 2.25], since we now know that an element $y$ is divisible by $x^{n}$ if and only if the first $n$ terms of its series $\theta(y)$ vanish. Since $m_{0}$ was arbitrary, this shows that $x$ is weakly almost prime, finishing the proof of assertion (vi).

We finally consider assertion (vii). We have already noted that $(\lambda-X)$ is invertible in $\mathcal{A}_{0}[[X]]$ if $\lambda \neq 0$. This shows that for every $\lambda \neq 0$ there exists $u_{\lambda}$ in $\mathcal{A}^{\sharp}$ such that

$$
(\lambda-x) u_{\lambda} \in 1+\bigcap_{n=1}^{\infty} x^{n} \mathcal{A}
$$


so, certainly,

$$
(\lambda-x) u_{\lambda} \in 1+\overline{\bigcap_{n=1}^{\infty} x^{n} \mathcal{A}} .
$$

Let a "dot" denote the coset in the Fréchet algebra $\mathcal{A}^{\sharp} / \overline{\bigcap_{n=1}^{\infty} x^{n} \mathcal{A}}$. It follows that $(\lambda-\dot{x}) \dot{u}_{\lambda}=\dot{i}$ for every $\lambda \neq 0$, so that $\sigma(\dot{x})=\{0\}$.

Suppose that for some seminorm $\|\cdot\|_{n}$ we have

$$
x^{k} \notin{\overline{x^{k+1} \mathcal{A}^{\sharp}}}^{n}
$$

for every $k \in \mathbf{N}$. Let $\pi_{n}$ be the canonical projection of the Arens-Michael isomorphism, and let $\mathcal{B}_{n}$ be the corresponding $n$th Banach algebra (on which the $\|\cdot\|_{n}$ seminorm is a norm). Since $\pi_{n}$ has dense range, it must also be the case that

$$
\left(\pi_{n}(x)\right)^{k} \notin \overline{\left(\pi_{n}(x)\right)^{k+1} \mathcal{B}_{n}}
$$

for every $k \in \mathbf{N}$ (the closure being taken in the Banach algebra $\mathcal{B}_{n}$ ). For each $k \in \mathbf{N}$ we can choose a continuous linear functional $\varphi_{k}$ in the dual of $\mathcal{B}_{n}$ with $\varphi_{k}\left(\left(\pi_{n}(x)\right)^{k}\right)=1$, but

$$
\varphi_{k}\left(\overline{\left(\pi_{n}(x)\right)^{k+1} \mathcal{B}_{n}}\right)=\{0\} .
$$

For each $k \in \mathbf{N}$ we can also choose a scalar $\lambda_{k}$ in the complex field sufficiently large such that

$$
\left|\lambda_{k}\right|>\left(\sum_{i=1}^{k-1}\left|\lambda_{i}\right|\left\|\varphi_{k}\right\|\left\|\left(\pi_{n}(x)\right)^{i}\right\|\right)+k\left\|\varphi_{k}\right\| .
$$

Since the homomorphism $\theta$ is onto, we can find an element $a \in \mathcal{A}^{\sharp}$ satisfying $\theta(a)=\sum_{i=1}^{\infty} \lambda_{i} X^{i}$ and for each $k \in \mathbf{N}$ there must also exist $b_{k} \in \mathcal{A}^{\sharp}$ such that $\theta\left(b_{k}\right)=\sum_{i=k+1}^{\infty} \lambda_{i} X^{i}$. Since $b_{k}$ is divisible by $x^{k+1}$ modulo the kernel of $\theta$, we have

$$
b_{k} \in x^{k+1} \mathcal{A}^{\sharp}+\left(\bigcap_{m=1}^{\infty} x^{m} \mathcal{A}\right) \subseteq{\overline{x^{k+1} \mathcal{A}^{\sharp}}}^{n} .
$$

This forces $\varphi_{k}\left(\pi_{n}\left(b_{k}\right)\right)=0$. Since

$$
a \in \lambda_{k} x^{k}+\sum_{i=0}^{k-1} \lambda_{k} x^{i}+b_{k}+\left(\bigcap_{m=1}^{\infty} x^{m} \mathcal{A}\right),
$$

we have that

$$
\begin{gathered}
\left\|\varphi_{k}\right\|\|a\|_{n} \geq\left|\varphi_{k}\left(\pi_{n}(a)\right)\right| \\
\geq\left|\lambda_{k}\right|\left|\varphi_{k}\left(\left(\pi_{n}(x)\right)^{k}\right)\right|-\sum_{i=1}^{k-1}\left|\lambda_{i}\right|\left|\varphi_{k}\left(\left(\pi_{n}(x)\right)^{i}\right)\right| \\
\geq\left|\lambda_{k}\right| \cdot 1-\sum_{i=1}^{k-1}\left|\lambda_{i}\right|\left\|\varphi_{k}\right\|\left\|\left(\pi_{n}(x)\right)^{i}\right\|>k\left\|\varphi_{k}\right\|
\end{gathered}
$$

for all $k \in \mathbf{N}$. Since each $\varphi_{k}$ is a nonzero functional, this forces $\|a\|_{n}$ to be infinite, a contradiction. Lemma 2.3 then shows that $\dot{x}$ must be locally nilpotent.

Suppose that some power $x^{m_{0}}$ is contained in $\overline{\bigcap_{n=1}^{\infty} x^{n} \mathcal{A}}$. Since

$$
x^{m_{0}} \in \overline{\bigcap_{m=1}^{\infty} x^{m} \mathcal{A}} \subseteq \overline{x^{m_{0}+1} \mathcal{A}^{\sharp}} \subseteq \overline{x^{m_{0}} \mathcal{A}^{\sharp}},
$$


this means that $x$ has finite closed descent, which is the first alternative of (vii). Suppose that $x$ is locally nilpotent and let $n \in \mathbf{N}$. Then there exists $m \in \mathbf{N}$ such that $\left\|x^{m}\right\|_{n}=0$. This means that

$$
x^{m_{0}} \in \overline{\bigcap_{k=m}^{\infty} x^{k} \mathcal{A}^{\sharp}} \subseteq \mathcal{I}_{n} .
$$

Since $n$ was arbitrary, we conclude that $x^{m_{0}} \in \mathcal{I}_{n}$ for all $n \in \mathbf{N}$, and that $x$ must be nilpotent, a contradition to (i). Hence $x$ must be nonlocally nilpotent if some power $x^{m_{0}}$ is contained in $\overline{\bigcap_{n=1}^{\infty} x^{n} \mathcal{A}}$.

Then if $\mathcal{A}^{\sharp}$ were a commutative radical Fréchet algebra with identity adjoined, Corollary 2.5 would imply that $x$ is not weakly almost prime, contradicting assertion (vi) proved above. Therefore, if the first assertion of (vii) holds, it must be the case that $\mathcal{A}^{\sharp}$ cannot be a commutative radical Fréchet algebra with identity adjoined.

If $x$ does not have finite closed descent, we have already shown that $\dot{x}$ is locally nilpotent, and that $\sigma(\dot{x})=\{0\}$. If $\mathcal{A}$ were a Banach algebra, then $\dot{x}$ would have to be nilpotent; that is,

$$
x^{m_{0}} \in \overline{\bigcap_{m=1}^{\infty} x^{m} \mathcal{A}}
$$

for some $m_{0} \in \mathbf{N}$. However, this forces $x$ to have finite closed descent as we noted above. Consequently, in the second alternative of (vii) it must be the case that $\mathcal{A}$ is not a Banach algebra and $\dot{x}$ is locally nilpotent but non-nilpotent. This finishes the proof of (vii) and the proof of the proposition.

\section{§3. Derivations on Radical Fréchet Algebras with Identity Adjoined}

In this section we investigate how deriviations can lead to local power series quotients.

Definition 3.1. A derivation on a (possibly noncommutative) Fréchet algebra $\mathcal{A}$ is a linear map $D$ from $\mathcal{A}$ to itself satisfying

$$
D(a b)=a(D b)+(D a) b
$$

for all $a, b$ in $\mathcal{A}$. We emphasize that we do not require $D$ to be continuous.

Note that a derivation $D$ extends to $\mathcal{A}^{\sharp}$ in an obvious way by defining (if necessary) $D 1=0$. We now state a well-known result from the theory of automatic continuity.

Lemma 3.2. Let $D$ be a (possibly discontinuous) derivation on a Fréchet algebra $\mathcal{A}$. Let $I$ be a closed ideal of $\mathcal{A}$ and let $Q_{I}$ denote the continuous canonical quotient map from $\mathcal{A}$ onto $\mathcal{A} / I$. For each $k \in \mathbf{N}$ define the separating subspace of $D^{k}$ as

$$
\mathcal{S}\left(D^{k}\right) \equiv\left\{z \in \mathcal{A} \mid \exists x_{i} \rightarrow 0 \text { in } \mathcal{A} \text { and } D^{k}\left(x_{i}\right) \rightarrow z\right\} .
$$

If the codimension $[\mathcal{A}: I]$ is finite, then the following are equivalent:

(i) $Q_{I} D^{k}$ is continuous on $I$ for all $k \in \mathbf{N}$;

(ii) $Q_{I} D^{k}$ is continuous on $\mathcal{A}$ for all $k \in \mathbf{N}$;

(iii) $\mathcal{S}\left(D^{k}\right) \subseteq I$ for all $k \in \mathbf{N}$.

In the special case where $\mathcal{A}$ is actually a Banach algebra, any one of the above implies the following condition:

(i) If $I$ is a primitive ideal of $\mathcal{A}$, then $D(I) \subseteq I$. 
Proof. Assume that condition (i) holds. Since a finite extension of a continuous linear mapping on a closed subspace is continuous, it is clear that condition (ii) must hold also. Clearly condition (ii) implies condition (i). Hence they are equivalent. Assume that condition (ii) holds. It is fundamental (see Thomas1, discussion on results (1)-(3), pages 518-519]) that $Q_{I} D^{k}$ will be continuous on $\mathcal{A}$ precisely when $Q_{I}\left(\mathcal{S}\left(D^{k}\right)\right)=\{0\}$. This will happen precisely when $\mathcal{S}\left(D^{k}\right) \subseteq I$. Hence, condition (ii) is equivalent to condition (iii).

Suppose $\mathcal{A}$ is a Banach algebra and assume that condition (ii) above holds. An application of [Thomas3, Lemma 1.1] shows that there exists a constant $C>0$ such that

$$
\left\|Q_{I} D^{k}\right\| \leq C^{k}
$$

for all $k \in \mathbf{N}$ (it is not necessary for $I$ to be primitive for this). Now, given that $I$ is primitive, apply [Thomas3, Lemma 1.2] and this shows that $D(I) \subseteq I$.

It is natural to ask what the structure of $D$ is like in the simplest possible (nontrivial) case; namely, a derivation $D$ on a commutative radical Fréchet algbra $\mathcal{R}^{\sharp} \cong \mathbb{C} \cdot 1 \oplus \mathcal{R}$ with identity adjoined. Here $\operatorname{rad}\left(\mathcal{R}^{\sharp}\right)=\mathcal{R}$, all elements $r \in \mathcal{R}$ have spectrum equal to $\{0\}$, and there is precisely one continuous strictly irreducible representation $\pi$ taking $(\lambda 1+r)$ to $\lambda$.

We are, of course, interested in the case where $\mathcal{R}$ is not invariant under $D$, and hence, by Thomas2], $\mathcal{R}^{\sharp}$ cannot be a Banach algebra. In addition, since $\mathcal{R}^{\sharp}$ is commutative, we can multiply $D$ by a suitable invertible element in order to obtain a new derivation which takes some element $x$ in the radical $\mathcal{R}$ to the identity 1 in $\mathcal{R}^{\sharp}$.

Recalling Definition 1.6, there is always a largest $x$-divisible subspace of $\mathcal{R}^{\sharp}$, which we will denote $\mathcal{D}_{x}$ and which is easily seen to lie in $\mathcal{R}$. By Thomas2 Lemma 2.8] the torsion subspace is $x$-divisible so that $\mathcal{D}_{x}=\bigcap_{m=1}^{\infty} x^{m} \mathcal{R}$. Since $\sigma(x)=\{0\}$, and since the difference, $(D(x \cdot)-x D(\cdot))$, is continuous, we can apply a basic result from the theory of automatic continuity on Fréchet spaces [Thomas1. Lemma 1.2] to conclude that for every $k \in \mathbf{N}$ there exists $n(k) \in \mathbf{N}$ such that

$$
{\overline{x^{m} \mathcal{S}(D)}}^{k}={\overline{x^{n(k)} \mathcal{S}(D)}}^{k} \subseteq{\overline{\mathcal{D}_{x}}}^{k}
$$

whenever $m \geq n(k)$. With this as motivation, we make the following definition.

Definition 3.3. With the commutative radical Fréchet algebra $\mathcal{R}^{\sharp}$, the (possibly discontinuous) derivation $D$, and the element $x \in \mathcal{R}$ as above, we define for each $k \in \mathbf{N}$ the set

$$
\mathcal{E}_{k} \equiv\left\{a \in \mathcal{R}^{\sharp} \mid x^{n} a \in{\overline{\mathcal{D}_{x}}}^{k} \text { for some } n \in \mathbf{N}\right\}
$$

and we let $\mathcal{E}$ denote the intersection of the closures

$$
\mathcal{E} \equiv \bigcap_{k=1}^{\infty} \overline{\mathcal{E}_{k}} .
$$

It is routine to check that $\left\{\mathcal{E}_{k}\right\}_{k=1}^{\infty}$ is a nonincreasing sequence of (not necessarily closed) ideals of $\mathcal{R}^{\sharp}$ and that

$$
\mathcal{E}_{k} \supseteq{\overline{\mathcal{D}_{x}}}^{k}={\overline{\bigcap_{m=1}^{\infty} x^{m} \mathcal{R}}}^{k} .
$$


If we let $Q_{k}$ be the canonical continuous quotient map from $\mathcal{R}^{\sharp}$ onto $\mathcal{R}^{\sharp} /{\overline{\mathcal{D}_{x}}}^{k}$, it is clear that $Q_{k} x^{n(k)} \mathcal{S}(D)=\{0\}$. Therefore $Q_{k} x^{n(k)} D$ is continuous, and for each $k \in \mathbf{N}$ we can find $\ell(k) \in \mathbf{N}$, larger than both $n(k)$ (so that $Q_{k} x^{\ell(k)} D$ is also continuous) and $k$ (so that ${\overline{\mathcal{D}_{x}}}^{\ell(k)} \subseteq{\overline{\mathcal{D}_{x}}}^{k}$ ), and $C_{k}>0$ such that

$$
\left\|Q_{k} x^{n(k)} D y\right\|_{k} \leq C_{k}\|y\|_{\ell(k)} \text {. }
$$

Since $D\left(\mathcal{D}_{x}\right) \subseteq \mathcal{D}_{x}$, it must also be true that $Q_{k} x^{n(k)} D\left(\mathcal{D}_{x}\right) \subseteq Q_{k} x^{n(k)} \mathcal{D}_{x}=\{0\}$. Therefore, from inequality (3.4), we see that

$$
Q_{k} x^{n(k)} D\left(\overline{\mathcal{D}}^{\ell(k)}\right)=\{0\}
$$

for $k=1,2, \ldots$ Let $a \in \mathcal{E}_{\ell(k)}$ such that there exists $n \in \mathbf{N}$ with $x^{n} a \in{\overline{\mathcal{D}_{x}}}^{\ell(k)}$. The Leibniz property shows that

$$
Q_{k} x^{n+n(k)} D a=Q_{k} x^{n(k)} D\left(x^{n} a\right)-Q_{k}\left(n x^{n(k)+n-1} a\right)=0-0=0
$$

using equation (3.5), and the fact that ${\overline{\mathcal{D}_{x}}}^{\ell(k)} \subseteq{\overline{\mathcal{D}_{x}}}^{k}$. This shows that $D \mathcal{E}_{\ell(k)} \subseteq \mathcal{E}_{k}$ for each $k \in \mathbf{N}$. Also, since $x^{n(k)} \mathcal{S}(D) \subseteq{\overline{\mathcal{D}_{x}}}^{k}$, we have $\mathcal{S}(D) \subseteq \mathcal{E}_{k}$ for each $k \in \mathbf{N}$. For each $k \in \mathbf{N}$ let $R_{k}$ be the canonical continuous quotient map from $\mathcal{R}^{\sharp}$ onto $\mathcal{R}^{\sharp} / \overline{\mathcal{E}_{k}}$. It is easily checked that $R_{k} \mathcal{S}(D) \equiv\{0\}$ and, hence, $R_{k} D$ is continuous for each $k \in \mathbf{N}$. Since $R_{k} D\left(\mathcal{E}_{\ell(k)}\right)=\{0\}$, it follows that $R_{k} D\left(\overline{\mathcal{E}_{\ell(k)}}\right)=\{0\}$. This shows that $D\left(\overline{\mathcal{E}_{\ell(k)}}\right) \subseteq \overline{\mathcal{E}_{k}}$ for all $k \in \mathbf{N}$. We are now ready to state our first lemma.

Lemma 3.6. With the commutative radical Fréchet algebra $\mathcal{R}^{\sharp}$, the (possibly discontinuous) derivation $D$, and the element $x \in \mathcal{R}$ as above, the set $\mathcal{E}$ is a closed ideal of $\mathcal{R}^{\sharp}$ with the following properties:

(i) $\mathcal{E}$ is D-invariant;

(ii) the separating ideal $\mathcal{S}(D)$ is contained in $\mathcal{E}$;

(iii) $D$ drops to a continuous derivation $\dot{D}$ from the quotient Fréchet algebra $\mathcal{R}^{\sharp} / \mathcal{E}$ to itself and $\mathcal{S}\left(D^{k}\right) \subseteq \mathcal{E}$ for all $k \in \mathbf{N}$.

Proof. It is clear that $\mathcal{E}$ is a closed ideal since each of the $\mathcal{E}_{k}$ 's is an ideal. Assertion (i) follows from the fact that $D\left(\overline{\mathcal{E}_{\ell(k)}}\right) \subseteq \overline{\mathcal{E}_{k}}$ for each $k \in \mathbf{N}$. Assertion (ii) follows from the fact that $\mathcal{S}(D) \subseteq \mathcal{E}_{k}$ for each $k \in \mathbf{N}$. If $R$ is the canonical continuous quotient map from $\mathcal{R}^{\sharp}$ onto $\mathcal{R}^{\sharp} / \mathcal{E}$, then $R \mathcal{S}(D)=\{0\}$ and hence $R D$ is continuous. Consequently, since $R D(\mathcal{E})=\{0\}$, it must be the case that $R D$ factors through $\mathcal{R}^{\sharp} / \mathcal{E}$ as $\dot{D} R$ where $\dot{D}$ is a continuous derivation from $\mathcal{R}^{\sharp} / \mathcal{E}$ into itself defined by $\dot{D}(a+\mathcal{E})=(D a+\mathcal{E})$ for $a \in \mathcal{R}^{\sharp}$. This shows the final assertion, since $\mathcal{S}\left(D^{k}\right) \subseteq \mathcal{E}$ if and only if $R D^{k}$ is continuous; but $R D^{k}=\dot{D}^{k} R$ for $k=2,3, \ldots$ and the maps on the right-hand side are all continuous.

At this point we are confronted with three possibilities (however, we will see later that option (ii) of Proposition 3.7 cannot occur). Reconsider Definitions 1.1 and 1.10 with regard to $\mathcal{R}^{\sharp} / \overline{\bigcap_{m=1}^{\infty} x^{m} \mathcal{R}}$.

Proposition 3.7. Let $\mathcal{R}^{\sharp}$ be a commutative radical Fréchet algebra with identity adjoined (so that $\mathcal{R}^{\sharp} \cong \mathbb{C} \cdot 1 \oplus \mathcal{R}$ and $\mathcal{R}=\operatorname{rad}\left(\mathcal{R}^{\sharp}\right)$ ). Let $D$ be a (possibly discontinuous) derivation from $\mathcal{R}^{\sharp}$ to itself and let $x \in \mathcal{R}$ satisfy $D x=1$. Then at least one of the following occurs:

(i) $\mathcal{S}\left(D^{k}\right) \subseteq \mathcal{R}$ for all $k \in \mathbf{N}$ and, if $\mathcal{R}$ is a Banach algebra, then $D(\mathcal{R}) \subseteq \mathcal{R}$. 
(ii) For some $k_{0} \in \mathbf{N}$ we have $\mathcal{S}\left(D^{k_{0}}\right) \nsubseteq \mathcal{R}, x$ is nonlocally nilpotent, and $x$ has finite closed descent in $\mathcal{R}$; i.e., there exists $m_{0} \in \mathbf{N}$ such that

$$
x^{m_{0}} \in \overline{x^{m_{0}+1} \mathcal{R}^{\sharp}}=\overline{\bigcap_{m=1}^{\infty} x^{m} \mathcal{R}}=\overline{\mathcal{D}_{x}} .
$$

(iii) For some $k_{0} \in \mathbf{N}$ we have $\mathcal{S}\left(D^{k_{0}}\right) \nsubseteq \mathcal{R}$, and if $\dot{x}$ denotes the coset $\dot{x}=$ $x+\overline{\bigcap_{m=1}^{\infty} x^{m} \mathcal{R}}$ in $\mathcal{R}^{\sharp} / \overline{\bigcap_{m=1}^{\infty} x^{m} \mathcal{R}}$, then $\dot{x}$ is locally nilpotent.

Proof. Suppose that assertion (i) holds and that $\mathcal{R}$ is a Banach algebra. Since $\mathcal{R}$ is a primitive ideal of $\mathcal{R}^{\sharp}$ of finite codimension, Lemma 3.2 assertion (iv) shows that $D(\mathcal{R}) \subseteq \mathcal{R}$.

Now assume that $\mathcal{S}\left(D^{k_{0}}\right) \nsubseteq \mathcal{R}$ for some $k_{0} \in \mathbf{N}$. Apply Lemma 3.6 assertion (iii). Since $\mathcal{S}\left(D^{k}\right) \subseteq \mathcal{E}$ for all $k \in \mathbf{N}$, it cannot be the case that $\mathcal{E} \subseteq \mathcal{R}$. But then $\mathcal{E}$ contains an invertible element, and, being a closed ideal, must contain the identity element 1 . This means that $1 \in \overline{\mathcal{E}_{k}}$ for all $k \in \mathbf{N}$. But if $\mathcal{E}_{k} \subseteq \mathcal{R}$, then $\overline{\mathcal{E}_{k}} \subseteq \mathcal{R}$ since the radical $\mathcal{R}$ is closed. It must therefore be the case that $1 \in \mathcal{E}_{k}$ for all $k \in \mathbf{N}$. Hence, for each $k \in \mathbf{N}$ there exists $m(k) \in \mathbf{N}$ such that

$$
x^{m(k)} \in{\overline{\mathcal{D}_{x}}}^{k} \text {. }
$$

At this point we consider the coset $\dot{x}=x+\overline{\bigcap_{m=1}^{\infty} x^{m} \mathcal{R}}$ in the quotient Fréchet algebra $\mathcal{R}^{\sharp} / \overline{\bigcap_{m=1}^{\infty} x^{m} \mathcal{R}}$. If $x$ is locally nilpotent, so is $\dot{x}$ and assertion (iii) holds. If $x$ is nonlocally nilpotent but $\dot{x}$ is nilpotent, then $x^{m_{0}} \in \overline{\bigcap_{m=1}^{\infty} x^{m} \mathcal{R}}$ for some $m_{0} \in \mathbf{N}$. Since $\mathcal{D}_{x}=\bigcap_{m=1}^{\infty} x^{m} \mathcal{R}$, the fact that

$$
x^{m_{0}} \in \overline{\mathcal{D}_{x}} \subseteq \overline{x^{m_{0}+1} \mathcal{R}^{\sharp}} \subseteq \overline{\bigcap_{m=1}^{\infty} x^{m} \mathcal{R}}
$$

shows that assertion (ii) holds.

Finally suppose that $x$ is nonlocally nilpotent and $\dot{x}$ is non-nilpotent. In this case, statement (3.8) as well as the fact that $\mathcal{D}_{x}=\bigcap_{m=1}^{\infty} x^{m} \mathcal{R}$, shows that for each $k \in \mathbf{N}$ we have

$$
\left\|x^{m(k)}+\overline{\bigcap_{m=1}^{\infty} x^{m} \mathcal{R}}\right\|_{k}=0 .
$$

This proves that $\dot{x}$ is locally nilpotent and establishes assertion (iii).

The paper [Thomas2] (which handles the Banach space case) outlines necessary modifications (on page 450) for Fréchet spaces, but is rather sketchy and does not demonstrate that case (ii) of Proposition 3.7 cannot occur. We give the full argument below.

First note that $\mathcal{R}^{\sharp} / \mathcal{D}_{x}=\mathcal{R}^{\sharp} / \bigcap_{m=1}^{\infty} x^{m} \mathcal{R}$ has a natural $p$-adic topology $\tau_{x}$ with local base at zero consisting of the sets of cosets

$$
\left\{x^{k} \mathcal{R}+\left(\bigcap_{m=1}^{\infty} x^{m} \mathcal{R}\right)\right\}_{k=1}^{\infty} .
$$

It should be emphasized that $\tau_{x}$ is definitely not a Fréchet space topology. The surprising fact is that finite closed descent (Proposition 3.7, case (ii)), or local nilpotency (Proposition 3.7, case (iii)), both imply statement (3.8) and this is sufficient to make the topology $\tau_{x}$ complete. 
Lemma 3.9. Let $\mathcal{R}^{\sharp}$ be a commutative radical Fréchet algebra with identity adjoined (so that $\mathcal{R}^{\sharp} \cong \mathbb{C} \cdot 1 \oplus \mathcal{R}$ and $\mathcal{R}=\operatorname{rad}\left(\mathcal{R}^{\sharp}\right)$ ). Let $x \in \mathcal{R}$ satisfy $\mathcal{D}_{x}=$ $\bigcap_{m=1}^{\infty} x^{m} \mathcal{R}$. Suppose that for every $i \in \mathbf{N}$ there is $m(i) \in \mathbf{N}$ such that

$$
x^{m_{i}} \in{\overline{\mathcal{D}_{x}}}^{i}
$$

(this holds in cases (ii) and (iii) of Proposition 3.7). Let $\left\{a_{i}\right\}_{i=1}^{\infty}$ be any sequence of coefficients chosen from $\mathcal{R}^{\sharp}$ to form the formal series $\sum_{i=m_{1}}^{\infty} a_{k} x^{k}$. Then there exists an element $y_{1} \in{\overline{\mathcal{D}_{x}}}^{1}$ satisfying

$$
y_{1}-\left(\sum_{k=m_{1}}^{\left(m_{n+1}+(n-1)\right.} a_{k} x^{k}\right) \in x^{n} \overline{\mathcal{D}}_{x}^{n+1}
$$

for $n=1,2,3, \ldots$ Consequently, letting a "dot" denote the coset in the quotient Fréchet algebra $\mathcal{R}^{\sharp} / \bigcap_{m=1} \mathcal{R}$, the series $\sum_{k=m_{1}}^{\infty} \dot{a}_{k} \dot{x}^{k}$ converges to $\dot{y}_{1}$ in the natural p-adic topology $\tau_{x}$ on $\mathcal{R}^{\sharp} / \bigcap_{m=1}^{\infty} x^{m} \mathcal{R}$. Consequently, every absolutely summable series converges and $\left(\mathcal{R}^{\sharp} / \mathcal{D}_{x}, \tau_{x}\right)$ is complete.

Proof. Note that each closed ideal ${\overline{\mathcal{D}_{x}}}^{n}$ is a Fréchet space in the relative topology. For each $n \in \mathbf{N}$ define the affine maps $A_{n}:{\overline{\mathcal{D}_{x}}}^{n+1} \rightarrow{\overline{\mathcal{D}_{x}}}^{n}$ as follows:

$$
\begin{gathered}
A_{1} y=x y+\sum_{k=m_{1}}^{m_{2}} a_{k} x^{k}\left(\text { recall that } x^{m_{1}} \in \overline{\mathcal{D}}_{x}{ }^{1}\right), \\
A_{2} y=x y+\sum_{k=m_{2}+1}^{m_{3}+1} a_{k} x^{k-1}\left(\text { recall that } x^{m_{2}} \in{\overline{\mathcal{D}_{x}}}^{2}\right), \\
A_{3} y=x y+\sum_{k=m_{3}+2}^{m_{4}+2} a_{k} x^{k-2}\left(\text { recall that } x^{m_{3}} \in{\overline{\mathcal{D}_{x}}}^{3}\right), \\
A_{n} y=x y+\sum_{k=m_{n}+(n-1)}^{m_{n+1}+(n-1)} a_{k} x^{k-(n-1)}\left(\text { recall that } x^{m_{n}} \in \overline{\mathcal{D}}_{x}^{n}\right) .
\end{gathered}
$$

Note that $\sum_{m_{n}+(n-1)}^{m_{n+1}+(n-1)} a_{k} x^{k-(n-1)} \in{\overline{\mathcal{D}_{x}}}^{n}$ for $n=1,2, \ldots$. Since $x \mathcal{D}_{x}=\mathcal{D}_{x}$, we have

$$
{\overline{A_{n}{\overline{\mathcal{D}_{x}}}^{n+1}}}^{n} \supseteq{\overline{A_{n} \mathcal{D}_{x}}}^{n}={\overline{\mathcal{D}_{x}}}^{n}
$$

for $n=1,2, \ldots$. In addition, since each $A_{n}$ is affine, we have that

$$
\left\|A_{n} a-A_{n} b\right\|_{n+p}=\|x(a-b)\|_{n+p} \leq\|x\|_{n+p}\|a-b\|_{n+p}
$$

for all $a, b \in{\overline{\mathcal{D}_{x}}}^{n+1}$ and $p=0,1,2, \ldots$.

Lemma 2.2 above, with $C_{i}=\|x\|_{i}$ and $X_{n}={\overline{\mathcal{D}_{x}}}^{n}$, shows that the inverse, or projective, limit $P=\left\{\left(y_{n}\right)_{n=1}^{\infty} \mid A_{n} y_{n+1}=y_{n}\right.$ for $\left.n=1,2, \ldots\right\}$ is nonempty and that ${\overline{\pi_{n}(P)}}^{n}={\overline{\mathcal{D}_{x}}}^{n}$ (where $\pi_{n}$ is the $n$th coordinate projection) for $n=1,2, \ldots$. 
So, let $\left(y_{n}\right)_{n=1}^{\infty} \in P$ and compute

$$
\begin{gathered}
y_{1}=A_{1} y_{2}=x y_{2}+\sum_{k=m_{1}}^{m_{2}} a_{k} x^{k}, \\
y_{2}=A_{2} y_{3}=x y_{3}+\sum_{k=m_{2}+1}^{m_{3}+1} a_{k} x^{k-1} \text { and hence } y_{1}=x^{2} y_{3}+\sum_{k=m_{1}}^{m_{3}+1} a_{k} x^{k}, \\
y_{3}=A_{3} y_{4}=x y_{4}+\sum_{k=m_{3}+2}^{m_{4}+2} a_{k} x^{k-2} \text { and hence } y_{1}=x^{3} y_{4}+\sum_{k=m_{1}}^{m_{4}+2} a_{k} x^{k}, \\
y_{n}=A_{n} y_{n+1}=x y_{n+1}+\sum_{k=m_{n}+(n-1)}^{m_{n+1}+(n-1)} a_{k} x^{k-(n-1)}, \\
\text { and hence } y_{1}=x^{n} y_{n+1}+\sum_{k=m_{1}}^{m_{n+1}+(n-1)} a_{k} x^{k} .
\end{gathered}
$$

This shows that

$$
y_{1}-\left(\sum_{k=m_{1}}^{m_{n+1}+(n-1)} a_{k} x^{k}\right) \in x^{n}{\overline{\mathcal{D}_{x}}}^{n+1}
$$

for $n=1,2, \ldots$. Note that if we had started with a different element $\left(y_{n}^{\prime}\right)_{n=1}^{\infty} \in$ $P$, we would have that $\left(y_{1}-y_{1}^{\prime}\right) \in \bigcap_{m=1}^{\infty} x^{m} \mathcal{R}$; so $\dot{y}_{1}=\dot{y}_{1}^{\prime}$ in $\left.\mathcal{R}^{\sharp} / \bigcap_{m=1}^{\infty} x^{m} \mathcal{R}\right)$. Therefore, the series $\sum_{k=m_{1}}^{\infty} \dot{a}_{k} \dot{x}^{k}$ converges to $\dot{y}_{1}$ in the natural $p$-adic topology $\tau_{x}$ on $\mathcal{R}^{\sharp} / \bigcap_{m=1}^{\infty} x^{m} \mathcal{R}$. The fact that completeness is equivalent to every absolutely summable series converging is well known. This finishes the proof of the lemma.

From this point on we can follow either Thomas2, Proposition 2.18 to Proposition 2.24] or [Zariski Lemma 4] almost verbatim to establish our main result.

Theorem 3.10. Let $\mathcal{R}^{\sharp}$ be a commutative radical Fréchet algebra with identity adjoined (so that $\mathcal{R}^{\sharp} \cong \mathbb{C} \cdot 1 \oplus \mathcal{R}$ and $\mathcal{R}=\operatorname{rad}\left(\mathcal{R}^{\sharp}\right)$ ). Let $D$ be a (possibly discontinuous) derivation from $\mathcal{R}^{\sharp}$ to itself and let $x \in \mathcal{R}$ be any element with $D x$ invertible in $\mathcal{R}^{\sharp}$. Then at least one of the following occurs:

(i) $\mathcal{S}\left(D^{k}\right) \subseteq \mathcal{R}$ for all $k \in \mathbf{N}$ and, if $\mathcal{R}$ is a Banach algebra, then $D(\mathcal{R}) \subseteq \mathcal{R}$.

(ii) $\mathcal{R}^{\sharp}$ has a formal power series quotient based at $x, x$ does not have finite closed descent, $\mathcal{R}$ is not a Banach algebra, and if $\dot{x}$ denotes the coset containing $x$ in the quotient Fréchet algebra $\mathcal{R}^{\sharp} / \overline{\bigcap_{m=1}^{\infty} x^{m} \mathcal{R}}$, then $\dot{x}$ is locally nilpotent (but non-nilpotent).

Proof. Since $\mathcal{R}^{\sharp}$ is commutative and $D x=u$ with $u$ invertible in $\mathcal{R}^{\sharp}$, we can replace $D$ by the derivation $\tilde{D}$ (where $\tilde{D}$ is defined by $\tilde{D}(v)=u^{-1} D(v)$ ). Note then that $\tilde{D}(x)=1$. We will now drop the "tilde" and write $D$ for $\tilde{D}$. We apply Proposition 3.7. If assertion (i) holds, we are done.

Now assume either assertion (ii) or assertion (iii) holds. Since statement (3.8) is then true, we have that $\left(\mathcal{R}^{\sharp} / \mathcal{D}_{x}, \tau_{x}\right)$ is complete as a consequence of Lemma 3.9. 
For $\dot{x} \in \mathcal{R}^{\sharp} / \mathcal{D}_{x}$ define

$$
\theta(\dot{a})=\sum_{k=0}^{\infty}\left(\frac{(-1)^{k} D^{k}(a) x^{k}}{k !}+\mathcal{D}_{x}\right)
$$

the series converging as a consequence of the completeness. It is easily checked that $\theta$ is both a unital algebra homomorphism and projection of $\mathcal{R}^{\sharp} / \mathcal{D}_{x}$ onto a unital subalgebra $\mathcal{A}_{0}$ of $\mathcal{R}^{\sharp} / \mathcal{D}_{x}=\mathcal{R}^{\sharp} / \bigcap_{m=1}^{\infty} x^{m} \mathcal{R}$. In addition, the kernel of $\theta$ is precisely $\dot{x}\left(\mathcal{R}^{\sharp} / \mathcal{D}_{x}\right)$, and $\dot{D} \circ \theta=0$. We have already remarked that the derivation $D$ leaves $\mathcal{D}_{x}$ invariant; so it drops to a derivation $\dot{D}$ from $\mathcal{R}^{\sharp} / \mathcal{D}_{x}$ to itself. One then defines an algebra homomorphism

$$
\dot{a} \rightarrow \sum_{n=0}^{\infty}\left(\frac{\theta\left(\dot{D}^{n}(a)\right)}{n !} X^{k}\right)
$$

from $\mathcal{R}^{\sharp} / \mathcal{D}_{x}$ into $\mathcal{A}_{0}[[X]]$. It is clear that this homomorphism is onto. The fact that the kernel of $\theta$ is $\dot{x}\left(\mathcal{R}^{\sharp} / \mathcal{D}_{x}\right)$, together with $D x^{n}=n x^{n-1}$ for all $n \in \mathbf{N}$ show that the homomorphism is injective.

Finally, apply Proposition 2.6(vii) and the fact that $\mathcal{R}^{\sharp}$ is a commutative radical Fréchet algebra with identity adjoined to show that the first alternative is not possible and that the second alternative must hold (that is, $x$ cannot have finite closed descent, $\mathcal{R}$ is not a Banach algebra, and $\dot{x}$ is locally nilpotent but non-nilpotent). This demonstrates that assertion (iii) rather than assertion (ii) of Proposition 3.7 must have held, and finishes the justification of option (ii) of our current theorem.

At one time the author believed that condition (i) of Theorem 3.10 was true for both Fréchet algebras and Banach algebras. A recent example in Read2 shows that there exists a radical Fréchet algebra $\mathcal{R}$ of formal power series with a locally nilpotent indeterminate $X$ which has a discontinuous derivation $D$ on its unitization $\mathcal{R}^{\sharp}$ such that $D(X)=1$ with the separating ideal $\mathcal{S}(D)$ equal to the entire algebra $\mathcal{R}^{\sharp}$. This example shows that condition (i) may fail for a Fréchet algebra, and hence condition (ii) is not vacuous.

\section{REFERENCES}

[Allan1] Allan, G. R., Embedding the algebra of all formal power series in a Banach algebra, Proc. London Math. Soc., (3) 25 (1972), 329-340. MR 46:4201

[Allan2] Allan, G. R., Fréchet algebras and formal power series, Studia Math., 119 (3) (1996) 271-288. MR 97f:46079

[Arens] Arens, R. F., A generalization of normed rings, Pacific J. Math., 2 (1952), 455-471. MR 14:482b

[Bonsall-Duncan] Bonsall, F. F., and Duncan, J. Complete Normed Algebras, SpringerVerlag, 1973. MR 54:11013

[Dales-McClure] Dales, H. G. and McClure, J. P., Higher point derivations on commutative Banach algebras, II, J. London Math. Soc., (2), 16 (1977) 313-325. MR 57:13498b

[Esterle] Esterle, J., Mittag-Leffler methods in the theory of Banach algebras and a new approach to Michael's problem, in Contemp. Math. 32, Amer. Math. Soc., 1984, 107-129. MR 86a:46056

[Johnson] Johnson, B. E., Continuity of derivations on commutative algebras, Amer. J. Math., 91 (1969) 1-10. MR 39:7433

[Johnson-Sinclair] Johnson, B. E. and Sinclair, A. M., Continuity of derivations and a problem of Kaplansky, Amer. J. Math., 90 (1968) 1067-1073. MR 39:776 
[Loy] Loy, R. J., Multilinear mappings and Banach algebras. J. London Math. Soc., (2) 14 (1976) 423-429. MR 56:12890]

[Michael] Michael, E. A., Locally multiplicatively-convex topological algebras, Mem. Amer. Math. Soc. 11, (1952; third printing 1971). MR 14:482a

[Read1] Read, C. J., All primes have closed range, J. London Math. Soc., 33 (2001), 341-346. MR 2002e: 46050

[Read2] Read, C. J., Derivations on Fréchet algebras with large separating subspaces, preprint.

[Rickart] Rickart, C. E., General Theory of Banach Algebras, The University Series in Higher Mathematics, van Nostrand, Princeton, NJ, 1960. MR 22:5903

[Sinclair] Sinclair, A. M., Automatic continuity of linear operators, London Math. Soc. Lecture Notes, 21, Cambridge. Univ. Press, Cambridge, 1976. MR 58:7011

[Thomas1] Thomas, M. P., Automatic continuity for linear functions intertwining continuous linear operators on Fréchet spaces, Canad. J. Math. 30, no. 3, (1978) 518-530. MR 58:2398

[Thomas2] Thomas, M. P., The image of a derivation is contained in the radical, Annals of Math., 128 (1988) 435-460. MR 90d:46075

[Thomas3] Thomas, M. P., Primitive ideals and derivations on noncommutative Banach algebras, Pacific J. Math., 159, no. 1 (1993) 139-152. MR 94e:46087

[Thomas4] Thomas, M. P., Elements in the radical of a Banach algebra obeying the unbounded Kleinecke-Shirokov conjecture, E. Albrecht and M. Mathieu, Banach Algebras '97 (Blaubeuren), de Gruyter, Berlin, 1998, 475-487. MR 99j: 46055

[Villena] Villena, A. R., Derivations on Jordan-Banach algebras, Studia Math., 118 (3) (1996) 205-229. MR 97c:46059

[Zariski] Zariski, Oscar, Studies in Equisingularity I. Equivalent singularities of plane algebroid curves, Amer. J. Math., 87 (1965) 507-536. MR 31:2243

Department of Mathematics, California State University at Bakersfield, BakersFIELD, CALIFORNIA 93311

E-mail address: marc@cs.csubak.edu 